\title{
The search for the fundamental QCD string in anti de Sitter space
}

\section{Richard C. Brower*}

Physics Department, 590 Commonwealth Ave, Boston University, Boston, MA 02215,USA

E-mail: browerabu. edu

The (original) flat space "hadronic" string discovered prior to QCD had both dramatic successes and equally dramatic failures. This talk will show how Maldacena's string/gauge duality conjecture offers a way out of the major failures. New QCD-like duals exhibit confinement and deconfinement at finite temperature, a qualitatively reasonable glueball and extended flux tube spectra as well as a domain wall mechanism for chiral symmetry breaking at strong coupling. Recently the hard components responsible for power behavior at wide angles and a unified treatment of soft (Regge)/hard(BFKL) Pomeron in high energy diffractive scattering has been identified at strong coupling. Lattice computations promise to provide more and more stringent "experimental" data to guide and test constructions of the fundamental QCD string.

XXIV International Symposium on Lattice Field Theory

July 23-28 2006

Tucson Arizona, US

${ }^{*}$ Speaker. 


\section{Introduction}

I wish to address the conjecture that

QCD is exactly equivalent to a fundamental string theory.

Paradoxically this old conjecture, with a somewhat checkered past, predates even the discovery of QCD itself (see Fig. 1 below). In the late 60's strong interaction scattering (or the hadronic S matrix) exhibited striking new features not observed in the best know quantum field theories of the day. The result was a systematic attempt to replace local quantum field theory with a new theory for a self-consistent relativistic quantum $\mathrm{S}$ matrix. This is the so called bootstrap program, which led to the discovery of string theory.

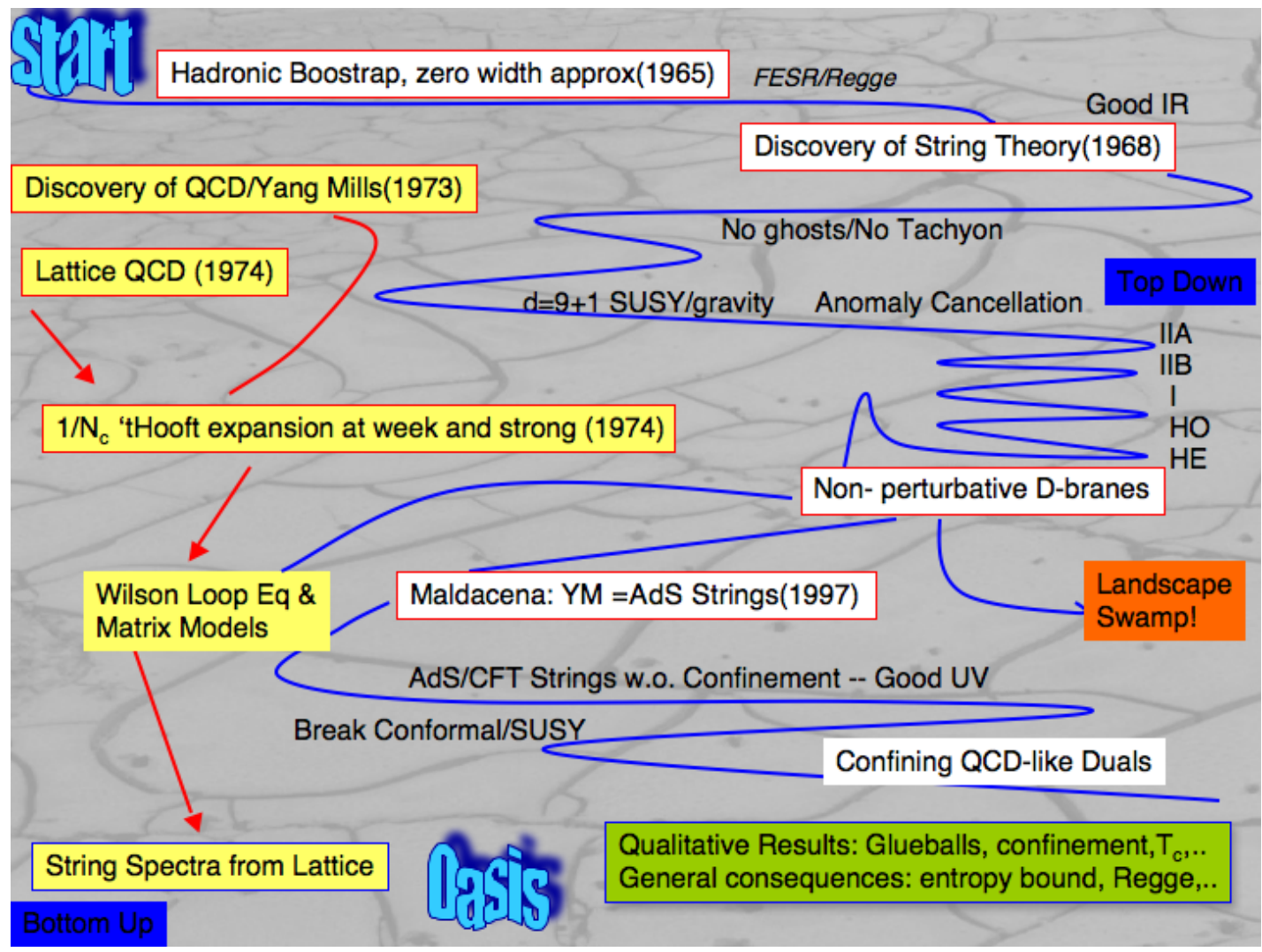

Figure 1: Long march through the desert landscape in search of the QCD string.

Consequently with the benefit of hindsight, it is appropriate to regard the discovery of perturbative string theory in the late 60's as the first attempt at constructing the fundamental QCD string. Subsequently the idea of a fundamental QCD string was largely ignored because the dynamics of this first attempt failed to reproduce the short distance physics, which was critical to the identification of SU(3) Yang Mills theory as the correct theory of the strong interactions in the first place. Instead the appearance of the graviton in flat space string theory led to the consensus that fundamental strings apply, if at all, to near Planckian scales where quantum gravity becomes important. 
In this view the Yang-Mills field theories of the standard model are the low energy effective theories of a fundamental string theory at short distances.

Progress on the rigorous construction of string perturbation theory began with the proof of no ghosts, removal of tachyons, the identification of the critical dimension and anomaly cancellations. The result was the identification of 5 perturbative vacua of the superstring in 10-d Minkowski space. Gradually a study of non-perturbative effects such as solitonic objects called D branes revealed a web of dualities connecting these vacua. All these developments tended to demonstrate the selfconsistency of superstring theory in the critical dimension and more support to the assumption that Yang Mills theories were simply a low energy effective theory of a fundamental string. These fundamental strings had no more direct connection to the electric flux tube in the confining phase of QCD than to magnetic vortices in a super conducting material. A useful phenomenology but not a alternative to Yang Mills theory.

However in mid 90's the picture began to change. A better understanding of non-perturbative string theory led Maldacena [1] to conjecture an exact AdS/CFT dualities between superstring in Anti de Sitter space and $\mathscr{N}=4$ SUSY Yang Mills. Here Yang-Mills and the string dual co-exist as equivalent description at the same energy scale. This not only made it very likely that many string theories are exactly dual (i.e. equivalent) to 4-d Yang Mills theory but gave for confining duals a reasonable explanation of the earlier failures of the QCD string based on flat space string dynamics.

This lecture will begin by a brief description of success and failure of the QCD string from the late 60's to extract lessons and then consider these issues in the light of the Maldacena's AdS/CFT constructions. The purpose is to make it at least plausible that there are no longer any obvious obstructions prohibiting an exact duality between QCD and a fundamental string field theory. Of course to move beyond plausibility, one needs to find the specific world sheet sigma model for the perturbative (i.e. large $N_{c}$ ) QCD string and make quantitative comparisons with experimental or lattice data. Even without an explicit formulation of the QCD string, it is still possible with care to abstract a few universal features from QCD like confining Yang Mills duals that give new non-perturbative results for QCD. Also it is worth noting that there are now two approaches to the search for the QCD string. A "top down" approach that begins with a specific AdS/CFT for superstring and its SUSY duals Yang-Mills theory and then modifies them to break all unwanted symmetries until they hopefully "flow" down to a pure QCD fixed point. This is highly technical approach but it has the advantage of a relatively rigorous starting point. So far this program has lead to several examples of QCD like confining duals but not QCD itself. The other approach is the more traditional "bottom up" strategy. Here one tries to find an effective low energy sigma model description or even a local AdS field theory that has more and more of the phenomenology of QCD, hoping to find the correct action by a series of small steps. Perhaps with the help of experimental and lattice data, this may ultimately be the best way to proceed to discover the action for the QCD string.

Before proceeding let me make clear what is meant by an equivalence to a fundamental string. No one disputes the fact that QCD in the confining phase has flux tubes so that a phenomenologically stringy phase exists. This can account for some of the long distance physics but by itself is not sufficient to the present task. An equivalence between two theories implies that there are two different classical Lagrangians whose quantum path integral leads to identical S matrix elements. The prototypical example of such an identity (often referred to as duality) is the 
equivalence between the quantum sine-Gordon theory and the massive Thirring model $[2,3]$.

$$
V(\phi)=\cos (\beta \phi / 2 \pi)
$$

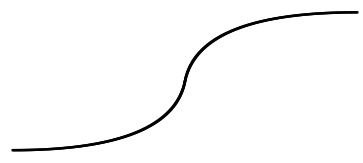

$$
g=\frac{1}{\beta^{2}}-\pi
$$

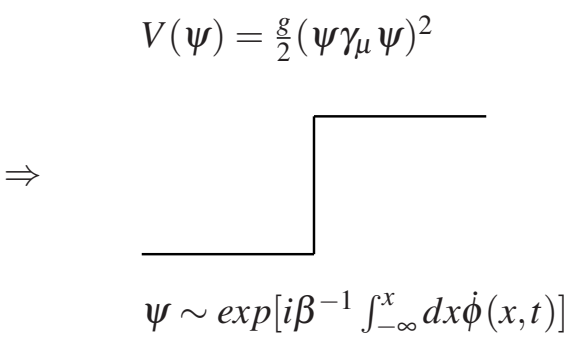

Figure 2: The classical sine-Gordon kink (lump) is represented by a point defect or local fermion field, $\psi(x)$, in the massive Thirring theory.

What lessons do we learn from this (and similar) examples? A semi-classical solitonic object can be a guide to this duality. In one theory the solitons with finite width (e.g. sine-Gordon kink) roughly correspond in the other theory to an exactly local objects (e.g. fermions fields in the Thirring model). In general one can identifies the degrees of freedom of the dual by taking an extreme (strong coupling) limit. However this correspondence is not simply a change of integration variables in the functional integral for the partition function. Both theories are treated quantum mechanically. This particular 2-d example of an exact duality between two quantum field theories has now become a familiar story for many 4-d Yang Mills theories as well. However the present search for a dual to QCD has some unusual features. We are not seeking an alternative local field theory by looking at strong coupling. Instead the extreme limit needed to identify the noninteracting solitonic objects for the dual theory is 'tHooft's large $N_{c}$ limit for the $S U\left(N_{c}\right)$ Yang-Mills theory at fixed "tHooft coupling $g^{2} N_{c}$. Also the dual variables are not zero dimensional (particle like) solitons but extended one dimensional strings. The combined large $N_{c}$ and strong coupling limit is the low energy limit of string theory or classical gravity does not have the full content of the dual theory.

\section{Lessons from the Past}

In this short talk it is impossible to trace even roughly the history of the search for the QCD string as depicted in Fig. 1 by paths wandering in the parched desert landscape of theory space. However one should appreciate two broad trends. The discovery of strings was based on empirical data on the hadronic scattering matrix. This was followed soon by the discovery of the Yang-Mills theory for QCD and by strings theory becoming an independent, not necessarily related, discipline. Consequently QCD physics (left side of figure) occasionally tried to justify (or derive) the string picture while string theory (right side of figure) became less and less to resemble hadronic physics. We begin with a few observations on this early period.

\subsection{Phenomenological Origins}

The discovery of string theory in the late 1960's followed from a detail study of the phenomenology of hadronic scattering, specifically finite energy sum rules constrained by Regge the- 
ory at high energies. For example the Regge limit for pion elastic scattering amplitude $\left(\pi^{+} \pi^{-} \rightarrow\right.$ $\pi^{+} \pi^{-}$) was traditionally parameterized as

$$
A_{\pi^{+} \pi^{-} \rightarrow \pi^{+} \pi^{-}}(s, t) \simeq g_{o}^{2} \Gamma\left[1-\alpha_{\rho}(t)\right]\left(-\alpha^{\prime} s\right)^{\alpha_{\rho}(t)},
$$

in Mandelstam variable $s=\left(p_{1}+p_{2}\right)^{2}$ and $t=\left(p_{1}+p_{3}\right)^{2}$. Physical scattering takes place for $t \leq 0$. At positive $t$, the Regge form factor,expressed as a Gamma function, incorporates the $t$ channel poles for the cross channel $\rho$ exchange at $J=1$ and higher spins recurrences for $J>1$. Since the ratio for the $\rho$ 's width to its mass is a small parameter $\left(\Gamma_{\rho} / m_{\rho} \simeq 0.1\right)$, one sought a new perturbative expansions starting with a zero width approximation. Since Regge trajectories, $\alpha(t)$, were observed to be almost linear function of $t$, this zero width approximation was enforced for all resonant states by using an exactly linear rho trajectory, $\alpha(t)=\alpha^{\prime} t+\alpha_{0}$, so that resonance poles at integer $J=\alpha\left(\mathrm{m}^{2}\right)$ had real masses [4]. In 1968 Veneziano [5] made a disarmingly simple observation that exact $s, t$ crossing symmetry could be imposed by assuming an amplitude of the form,

$$
A_{\pi^{+} \pi^{-} \rightarrow \pi^{+} \pi^{-}}(s, t)=g_{o}^{2} \frac{\Gamma\left[1-\alpha_{\rho}(t)\right] \Gamma\left[1-\alpha_{\rho}(s)\right]}{\Gamma\left[1-\alpha_{\rho}(s)-\alpha_{\rho}(t)\right]},
$$

the so called dual resonance model. Here "dual" referred to Dolan-Horn-Schmid duality [6] which states that the sum over s-channel resonances poles interpolates the power behavior of the leading Regge pole exchange,

$$
\sum_{r} \frac{g_{r}^{2}(t)}{s-\left(M_{r}-i \Gamma_{r}\right)^{2}} \simeq \beta(t)\left(-\alpha^{\prime} s\right)^{\alpha(t)},
$$

which is easily derived for the dual pion scattering amplitude (2.2). The Regge limit follows from the Sterling's approximation as $s \rightarrow \infty$ and the resonance expansion from the integral representation for the Beta function,

$$
A_{\pi^{+} \pi^{-} \rightarrow \pi^{+} \pi^{-}}(s, t)=-g_{o}^{2} \alpha_{\rho}(t) \int_{0}^{1} d x x^{-\alpha_{\rho}(s)}(1-x)^{-1-\alpha_{\rho}(t)} .
$$

Expanding at small $x$ we get,

$$
\begin{aligned}
& A_{\pi^{+} \pi^{-} \rightarrow \pi^{+} \pi^{-}}(s, t)=-g_{o}^{2} \sum_{J=1}^{\infty} \frac{\left(\alpha_{\rho}(t)\right)\left(\alpha_{\rho}(t)+1\right) \cdots\left(\alpha_{\rho}(t)+J-1\right)}{(J-1) !} \int_{0}^{1} d x x^{-1-\alpha(s)+J} \\
& \Rightarrow g_{o}^{2} \sum_{J=1}^{\infty} \frac{A_{J}\left(\alpha^{\prime} t\right)}{\alpha_{\rho}(s)-J} \simeq g_{o}^{2} \Gamma\left(-1-\alpha_{\rho}(t)\right)\left(-\alpha^{\prime} s\right)^{\alpha_{\rho}(t)},
\end{aligned}
$$

where $A_{J}$ is a polynomial of order $J$.

Moreover the initial enthusiasm for this model included a striking feature of chiral symmetry. In the soft pion limit $p_{1} \rightarrow 0$, the Adler zero,

$$
A_{\pi^{+} \pi^{-} \rightarrow \pi^{+} \pi^{-}}(s, t)=g_{o}^{2}\left(1-\alpha_{\rho}(s)-\alpha_{\rho}(t)\right) \frac{\Gamma\left[1-\alpha_{\rho}(t)\right] \Gamma\left[1-\alpha_{\rho}(s)\right]}{\Gamma\left[2-\alpha_{\rho}(s)-\alpha_{\rho}(t)\right]} \sim \alpha^{\prime}(s+t) \rightarrow 0,
$$

is realized if we take the phenomenologically reasonable values for the rho trajectory intercept, $\alpha_{\rho}(0)=0.5$. Further work led to the N-point generalization in Neveu and Schwarz's seminal paper [7] entitled "Factorizable Dual Model of Pions". So Veneziano's amplitude turns out to 
be the 4-point function of the NS superstring -ignoring the conformal constraint on the Regge intercept $(\alpha(0)=1)$ and the dimension of space time $(D=10)$ which were not understood at the time. Within a very short time, it was realized that amplitudes with arbitrary numbers of external lines could be easily written down and perturbative unitarization carried out be imposing cutting rules. Unfortunately as we will explain this initial enthusiasm was largely a mirage as a starting point for the QCD string.

\subsection{Large $N_{c}$ Topology}

In a sense the modern era of the QCD string begins almost immediately after the discovery of QCD itself with 'tHooft analysis [8] of the large $N_{c}$ limit in 1974. The problem one faced was to understand how the picture of valence quarks attached to the strings of the dual resonance model might arise in QCD. Even assuming some non-perturbative mechanism for electric confinement, one must find a small parameter to explain the zero resonance width approximation.

Guided by the basic premise that the string theory for QCD might be identified as an extreme (or strong coupling) limit of Yang-Mills theory, one is left with a quandary. In fact the full quantum theory for QCD has no independent coupling constant because by dimensional transmutation (or breaking of conformal symmetry at zero mass for the quarks) this coupling is replaced by a fundamental mass scale, $\Lambda_{q c d}$. For SU(3) QCD the only free dimensionless parameters are the scaled quark masses, $m_{q} / \Lambda_{q c d}$, and the $\theta$ parameter. The weak coupling expansion for $\mathrm{QCD}$, is really a shorthand for the loop expansion in $\hbar$, which is of course of great use in the UV for large "energies", $E / \Lambda_{q c d}$, due to "asymptotic freedom".

'tHooft asked whether the inverse of the rank of the group for $S U\left(N_{c}\right)$ Yang-Mills theory could be used as a formal expansion parameter. Indeed he noticed that by fixing $g^{2} N_{c}$ the large $N_{c}$ expansion did act to decouple the quarks form the theory at order $1 / N_{c}$. To the extend that decay widths required the production of quark anti-quark pairs, one might view this a natural explanation for the zero width expansion.

Consequently he reorganized the loop expansion in $\hbar$, as an expansion in powers of $1 / N_{c}$ holding fixed the 'tHooft coupling $g_{Y M}^{2} N_{c}$ (or more precisely fixed $\Lambda_{q c d}$ ). The result was the famous topological restructuring of the loop expansion as sum over Riemann surfaces. Starting from the action,

$$
S=\frac{1}{g_{Y M}^{2}} \operatorname{Tr}\left[\left(\partial_{\mu} A_{v}-\partial_{v} A_{\mu}+i\left[A_{\mu}, A_{v}\right]\right)^{2}\right]+\frac{1}{g_{Y M}^{2}} \bar{\Psi}\left(\gamma_{\mu} \partial_{\mu}-i A_{\mu}\right) \Psi,
$$

and writing down Feynman diagrams in the "double line" form, we count factors of $1 / N_{c}$ :

$$
\begin{gathered}
\text { Gluon Loops : } \delta_{r}^{r}=N_{c} \Rightarrow O\left(N_{c}^{F}\right) \\
\text { Gluon \& Quark Prop : } g_{Y M}^{2}=g_{Y M}^{2} N_{c} \times \frac{1}{N_{c}} \Rightarrow O\left(N_{c}^{-E}\right), \\
\text { Vertices : } \frac{1}{g_{Y M}^{2}}=\frac{1}{g_{Y M}^{2} N_{c}} \times N_{c} \Rightarrow O\left(N_{c}^{V}\right) .
\end{gathered}
$$

Using Euler's theorem we see that the factors of $N_{c}$ for color loops (faces F), gluon/quark propagators (edges E), interactions (vertices V) and quark flavor loops (boundaries B) combine,

$$
N_{c}^{F-E+V-B}=N_{c}^{\chi}=N_{c}^{2-2 H-B},
$$


to classify the topology of the graph as function of the number of glueballs propagators (i.e. handles H) and the quark loops ( i.e. boundaries B).

Remarkably the zero width expansion has now revealed one of the salient features of string theory, the topological expansion of string space time histories in terms of the genus of the world sheet. On the other hand, this is still perturbation theory so the connection to confining flux tubes in space-time is not obvious. However the same topology expansion can be shown to hold on the lattice at strong coupling in the confining phase as well. Since the lattice strong coupling expansion is actually a sum over surfaces of electric flux , the physical mechanism for confinement is clearly string-like flux tubes, in spite of the breaking of Lorenz invariance due the lattice artifacts. The argument is analogous to weak coupling. For illustration consider the Wilson form of the pure gauge action,

$$
S=\frac{1}{g_{Y M}^{2}} \sum_{P} \operatorname{Tr}\left[2-U_{P}-U_{P}^{\dagger}\right] \quad, \quad U_{P}=U_{\mu}(x) U_{v}(x+\mu) U_{\mu}^{\dagger}(x+v) U_{v}^{\dagger}(x) \quad, \quad U_{\mu}=\exp \left[i a A_{\mu}\right] .
$$

as a sum over plaquettes. In strong coupling the action is expanded in a power series and each link variable $\left(U_{\mu}(x)\right)$ is integrated over its Haar measure. To get a non-zero result every link in the expansion must be paired with (at least) one anti-link $\left(U \rightarrow U^{\dagger}\right)$. This leads immediately to the rule:

$$
\begin{aligned}
\text { Plaquettes : } & \frac{1}{g_{Y M}^{2}}=\frac{1}{g_{Y M}^{2} N_{c}} \times N_{c} \Rightarrow O\left(N_{c}^{F}\right), \\
\text { Links : } & \int d U U_{r_{1}}^{l_{1}} U_{l_{2}}^{\dagger r_{2}}=\frac{1}{N_{c}} \delta_{l_{2}}^{l_{1}} \delta_{r_{1}}^{r_{2}} \Rightarrow O\left(N_{c}^{-E}\right), \\
\text { Sites }: & \delta_{r}^{r}=N_{c} \Rightarrow O\left(N_{c}^{V}\right) .
\end{aligned}
$$

Treating quark loops boundaries as before, Euler's theorem yields exactly the same topological result as in weak coupling (ignoring self-intersections of surfaces). However it should be realized that the meaning is quite different. The vertices give the index sums, the faces are now field strengths and edges are not propagators. Apparently the topology of large $N_{c}$ Yang Mills is a robust feature in need of a deeper explanation.

In a real sense the large $N_{c}$ limit if it exists can be considered as the definition the QCD string perturbative expansion order by order in the string coupling $g_{s} \sim 1 / N_{c}$. But to go beyond this theoretical assertion by explicitly take the large $N_{c}$ limit to give a mathematical tractable definition of the perturbative QCD string (even for the leading term at $N_{c}=\infty$ ) has proven frustrating, except for two dimensional QCD. For 2-d QCD, in pure glounic (closed string) sector, Gross and Taylor [9] have identified the underlying topological expansion in great detail. However in spite of many interesting efforts, much work remains to fully understand the 'tHooft's equation for the (open string) meson propagator as world sheet string theory. This will be discussed briefly in the conclusion.

Also it is interesting to note that there is more than one large $N_{c}$ limit [10]. One can choose to treat quark field as an anti-symmetric tensor, $\Psi^{i j}=\varepsilon^{i j k} \psi_{k}$ in color. If one now takes the large $N_{c}$ limit of 1 flavor QCD with this tensor representation for quark fields, the fermion loop is no longer subdominant. In fact the leading term in the bosonic sector can be shown to be precisely the same as the large $N_{c}$ limit of $\mathscr{N}=1$ SUSY Yang Mills theory! Should we be alarmed at this in view of the glib statement that the large $N_{c}$ limit defines string perturbation theory. I think not. In fact 
the full non-perturbative QCD string theory might well have more than one weak coupling string expansion, analogous to the now conventional view of superstrings in 10-d.

\subsection{Failures of flat space-time strings}

We should now take a break from this discourse, following the wandering paths on the right side of Fig. 1, and learn all of rules of superstring perturbations theory [11]. With the help of anomaly cancellation, we would discover 5 consistent perturbation expansions - free of tachyons and negative norm (i.e. ghost) states. But the resulting phenomenology for perturbative superstrings (in flat space-time) has 4 major disasters from the view point of a QCD string:

1. Zero mass states (i.e $1^{-}$gauge/ $2^{++}$graviton)

\section{Supersymmetry}

3. Extra dimension: $4+6=10$

\section{No Hard Scattering Processes}

One can easily imagine that the first 3 difficulties could be remedied by "forcing" some form of compactification of the extra 6 dimensions, breaking all unwanted symmetries. Indeed in view of the fact that superstrings include gravity, it is even natural to suppose that solutions should include non-trivial space-time geometries. However the 4th problem of no hard scattering reveals a fundamental mismatch between soft strings and hard partonic QCD. All in all an abject failure for QCD strings but an interesting framework for a theory of quantum gravity interacting with matter. A Theory Of Everything (TOE) perhaps. There are two possible consequence, either the fundamental QCD string has nothing to do with a fundamental superstring or there are dramatic new effects when non-trivial background metrics are considered.

\section{Lesson from AdS/CFT}

String theory has undergone a tremendous transformation in the last 35 years. In the "First String Revolution" the perturbative string vacua were restricted to five alternatives (IIA, IIB, I, H0, HE) by the requirement to cancel tachyons, ghosts and anomalies. This appeared to restrict dramatically the space of possible string theory. In the "Second String Revolution", non-perturbative dualities went on to relate these 5 cases (and M theory) into a single connected manifold. However, that is not the end of the story. Solitonic objects called D-branes have given rise to a tremendous explosion of possible vacua in non-trivial (warped) target spaces. The infrared the physics of strings in these non-trivial backgrounds were seen to mimic a plethora of effective fields theories.

\subsection{AdS/CFT correspondence for Superstrings}

In 1998 Maldacena [1] realized that at least under certain circumstances string theories in curved space might be exactly equivalent (i.e dual) to Yang Mills theory. The first examples envolved a duality between string propagating in an Anti de Sitter (AdS) space and a conformal field theory (CFT). As a consequence this class of String/Gauge dualities has come to be know by the 

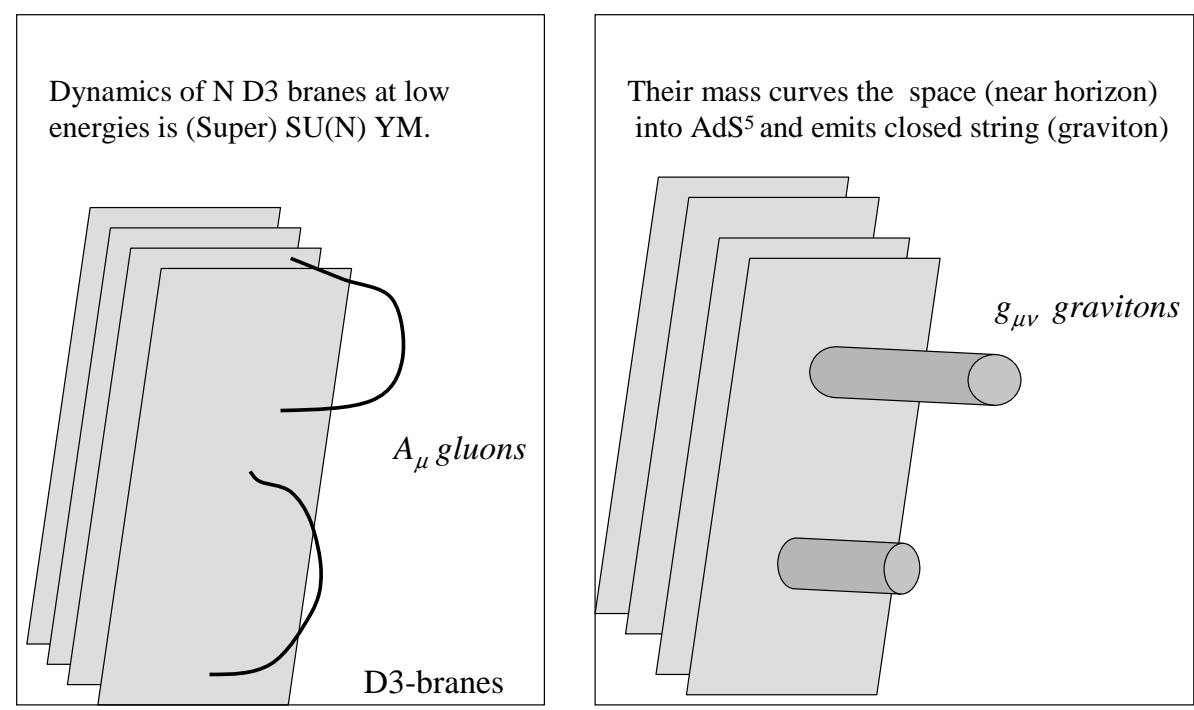

Figure 3: Open/closed string duality for $N_{c}$ D3 branes leading to the conjecture duality of IIB strings in $A d S^{5} \times S^{5}$ and $\mathscr{N}=4$ Super $\operatorname{SU}\left(N_{c}\right)$ Yang Mills theory.

phase "AdS/CFT" dualities. While technically all such AdS/CFT dualities are still unproven conjectures, the consistency relations are now so extensive that the existence of exact String/Gauge dualities in many special circumstances is hard to doubt.

Maldacena's first example consisted of IIB superstrings propagating in an $A d S^{5} \times S^{5} 10-\mathrm{d}$ manifold,

$$
d s^{2}=\frac{r^{2}}{R^{2}} \sum_{\mu=0}^{3} \eta_{\mu v} d x^{\mu} d x^{v}+\frac{R^{2}}{r^{2}}\left(d r^{2}+r^{2} d^{2} \Omega_{5}\right),
$$

which is dual to $4-\mathrm{d} \mathscr{N}=4$ Super $\mathrm{SU}\left(N_{c}\right)$ Yang Mills theory. The $\left(x_{\mu}, r\right)$ co-ordinates form the $A d S^{5}$ manifold with negative curvature with radius $R$ fibered by a $S^{5}$ sphere of (positive) radius $\mathrm{R}$ and metric $d^{2} \Omega_{5}$. The motivation for this duality is based on the background metric for a set of $N_{c}$ parallel massive D3 sources (see Fig. 3). Evidence had accumulated that there are two equivalent ways to model the dynamics of D3 branes. First by considering short open strings attached to the branes which at low energies is SUSY Yang Mills (SYM) theory and second by the near horizon fluctuations of closed IIB superstrings or at low energy IIB supergravity. The leap of faith was to conjecture that in the near horizon limit these two formulations are equivalent. In some sense this is a more refined version of the old open/closed duality found in the one loop unitarization of the open string scattering amplitude in flat space time. 
In this dual correspondence, the string (or gravity) correlation functions as you approach the boundary of $A d S^{5}(r \rightarrow \infty)$ are mapped into gauge invariant correlators in SYM theory. The discrete "Kaluza-Klein" modes in $S^{5}$ give the multiplets under SYM R symmetry $S U(5)$. By combining the subtle new idea of holography in $r$ and the more mundane Kaluza-Klein mechanism on $S^{5}$, we see how a 10-d string can be dual to a 4-d field theory. There is no loss of degrees of freedom. The 'tHooft gauge coupling is $g_{Y M}^{2} N_{c}=R^{4} / \alpha^{\prime 2}$, where the intrinsic string length scale is $\sqrt{\alpha^{\prime}}=l_{s}$. Consequently strong 'tHooft coupling gauge theory is dual to weak coupling gravity $\left(l_{s} \sim l_{\text {Planck }}\right)$ and the $1 / N_{c}$ expansion parameter is identified with the closed string coupling constant $g_{s}=g_{Y M}^{2} \sim$ $1 / N_{c}$ as one would expect from the large $N_{c}$ topological expansion. The isometries of the back ground are the conformal group $O(4,2)$ so that translations in $\log (r)$ act to remove the scale from the corresponding CFT. The 'tHooft coupling constant, $g_{Y M}^{2} N_{c}$ is a free parameter of the CFT.

Although the Maldacena string/gauge duality is believed to hold for general coupling and general $N_{c}$, it is difficult to quantize even free strings $\left(N_{c}=\infty\right)$ in this background which includes a non-zero Ramond-Ramond flux. In the strong coupling limit $\left(g_{s} \sim g_{Y M}^{2} \rightarrow \infty\right)$, the string tension diverges leaving only the center of mass motion of closed strings, which is equivalent to IIB gravity in the tree approximation. The weak coupling limit of classical gravity is easily solved. (Other special cases, such as the pp-wave limit, are tractable as well.)

One may view the correspondence in holographic terms. The Yang Mills UV (short distance) degrees of freedom are dual to excitations near to the AdS boundary at $r \rightarrow \infty$, while the IR (long distance physics) is represented by modes at small $r \rightarrow 0$. This mapping is referred to as IR/UV correspondence. A graphic illustration of this IR/UV correspondence is afforded by the scale breaking instanton solution to Yang-Mills located at $x^{\mu}$ with size $\rho$. This corresponds exactly to 0 -brane located at five dimensional co-ordinate $\left(x^{\mu}, r=R^{2} / \rho\right)$ in the $A d S^{5}$ manifold (see Fig. 4).

\subsection{Confinement}

Ironically this first example of Yang-Mills/String duality does not confine because the quantum field theory is exactly conformal. Wilson loops have pure Coulomb (rather than area law) behavior. When a large Wilson test loop is introduced on the boundary of AdS, the red shift factor $r^{2} / R^{2}$ of the metric allows the minimal surface area spanning the loop to remain finite by moving into the interior nearer and nearer to $r=0$. Hence the most well studies example of String/Gauge duality is not based on the narrow flux tubes and a linear interaction potential in the $\operatorname{IR}, V(L) \sim \alpha^{\prime} L$, at large distances), but strings that with a conformal interaction engery, $V(L) \sim 1 / L$, for all length scale). This is a major departure from earlier attempts at constructing string theory equivalent to large $N_{c}$ Yang Mills theory. Now we are starting from the opposite end of the problem with a string theory in AdS space that gives a good approximation to the Coulombic behavior of the gauge interaction of QCD at short distance! To look for models closer to QCD we must break conformal and supersymmetries and see how to obtain a linear potential with confinement at long distances.

These models invariably modify the metric in the IR, cutting it off at a finite value $r=r_{\text {min }}$. This IR cut-off plays the role of $\Lambda_{q c d}$. Two simple examples were suggested by Witten [12] by introducing a Euclidean AdS black hole background with a compact dimension (called $\tau$ ) whose radius set by the Hawking temperature: 
- $A d S^{5} \times S^{5}$ Black Hole for 10-d IIB string theory

- $A d S^{7} \times S^{4}$ Black Hole for 10-d IIA string theory

Both metrics have the general form,

$$
d s^{2}=\frac{r^{2}}{R^{2}} \eta_{\mu v} d x^{\mu} d x^{v}+\frac{R^{2}}{r^{2}\left[1-\left(r_{\min } / r\right)^{d}\right]} d r^{2}+\frac{r^{2}}{R^{2}}\left[1-\left(r_{\min } / r\right)^{d}\right] d \tau^{2}+d s_{X}^{2}
$$

asymptotically $A d S^{d+1}$ in the UV. The horizon of the black hole introduces a scale breaking cut-off, which we can identify roughly with $\Lambda_{q c d}=1 / r_{\min }$ or as we will see subsequently the scale of the glueball mass in strong coupling.

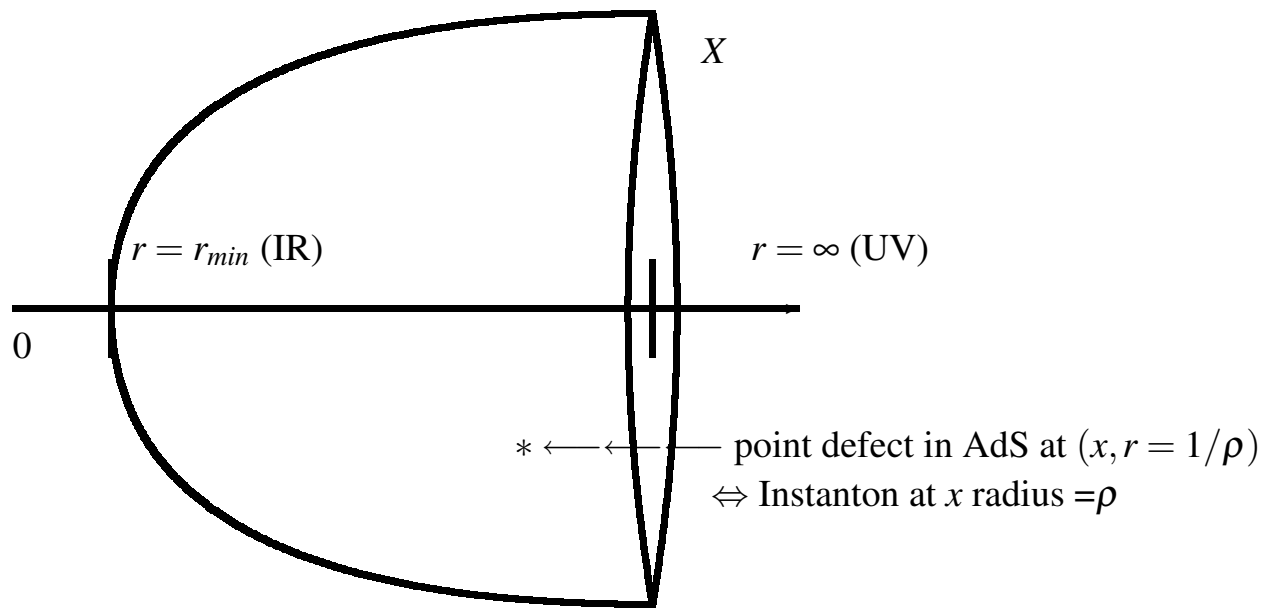

Figure 4: Deformed $A d S^{5}$ manifold with IR cut-off at $r=r_{\min }$ to give confinement for the dual Yang Mills theory.

In these black hole metrics, the minimal area surface spanning a Wilson loop of increasing size eventually must approach $r=r_{\text {min }}$. At this point the area of the surface no longer has a red shift factor and it grows proportional to the physical area of the Wilson loop itself. For example in the $A d S^{5}$ black hole the proper areas grows proportional to $r_{\min }^{2} / R^{2}$ giving a QCD tension $T_{q c d}=$ $1 / 2 \pi \alpha_{q c d}^{\prime}$ or Regge slope,

$$
\alpha_{q c d}^{\prime} \sim \alpha^{\prime} R^{2} / r_{\min }^{2} \sim \Lambda_{q c d}^{2} / \sqrt{g_{Y M}^{2} N_{c}}
$$

\section{Spectral Results from Confining Duals}

Based on the conformally broken backgrounds using Maldacena string/gauge duality, we can begin to do some calculation in QCD like theories, at least in the strong coupling limit. This is still far from the hoped for discovery of the QCD string. We are in the position somewhat similar to a lattice cut-off theory. The strong coupling limit brings along non-universal cut-off dependent effects. However unlike the lattice, we have (as yet) no algorithm (theoretical or numerical) to 
in principle send the cut-off to infinity. String/gauge duality presents a coupled problem, even in the large $N_{c}$. The world sheet sigma model for the string theory emits gravitons that perturb the background which in turn has a back reaction on the sigma model. Even finding the sigma model beta function perturbatively to the next order in $1 / \alpha^{\prime}$ is difficult. Still it is worth while to see if there is a reasonable spectrum in strong coupling approximation.

On the lattice side, where one can numerically take the weak coupling (continuum) limit, the spectra for glueballs [13] and the quantum states of a stretch string are becoming quit accurately determined [14]. Even extrapolating this spectra to the large $N_{c}$ limit has met with some success. In short the lattice has given and is capable of giving more accurate spectral data for the quantum QCD string. If it exists, there can be only one answer. This is a unique opportunity: A concrete string theory problem with copious "experimental" data to constrain its construction!
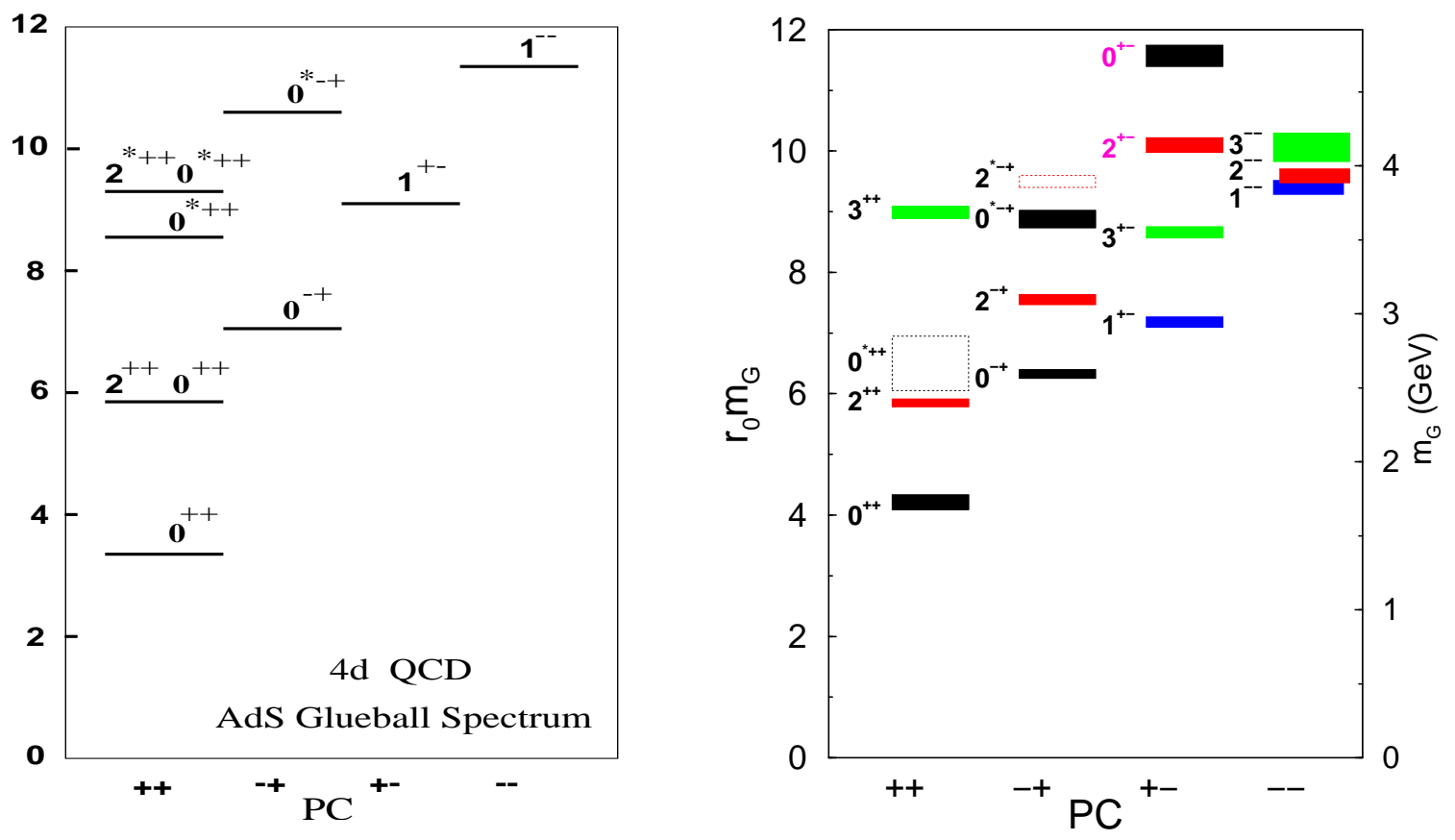

Figure 5: The AdS glueball spectrum from Brower,Mathur and Tan [15] for $Q C D_{4}$ in strong coupling (left) compared with the lattice spectrum of Morningstar and Peardon [13] for pure SU(3) QCD (right). The AdS cut-off scale is adjusted to set the lowest $2^{++}$tensor state to the lattice results in units of the hadronic scale $1 / r_{0}=410 \mathrm{Mev}$.

\subsection{Glueball Spectra}

The first such lattice AdS/CFT comparison was the computation of the strong coupling glueball spectrum in the $A d S^{7} \mathrm{M}$-theory black hole. The correspondence for the quantum numbers for the gravity modes in terms of the Yang-Mill fields are read off from the effective Born-Infeld action 
on the brane,

$$
S=\int d^{5} x \operatorname{det}\left[G_{\mu v}+e^{-\phi / 2}\left(B_{\mu v}+F_{\mu v}\right)\right]+\int d^{4} x\left(C_{1} F \wedge F+C_{3} \wedge F+C_{5}\right) .
$$

The entire spectrum for all states in the QCD super selection sector are now known and can be compared with lattice data for $\mathrm{SU}(3)$. The comparison is rather encouraging considered as a first approximation (see Fig. 5). All the states are in the correct relative order and the missing states at higher $\mathrm{J}$ are a direct consequence of strong coupling which pushes the string tension to infinity. It appears plausible that the $A d S^{7}$ black hole phase at strong coupling is rather smoothly connected to the weak coupling (confined) fixed point of QCD. However it must be stated that there is no general understanding of how the metric will be deformed so that all the unwanted charged Kaluza-Klein states in the extra compact directions decouple. All attempts to find better background solutions to supergravity as a starting point for QCD have failed in this regard.

\section{Stretched String Spectra}

An even more direct probe of the 5-th dimension is provided by the spectrum of the string stretched between infinitely heavy sources (see Fig 6). From the AdS/CFT viewpoint, starting with the ends of the string separated by a small distance L, we are probing the short distance Coulomb regime. Then as we increase $\mathrm{L}$, the minimal surface moves into the interior probing more and more IR physics. Finally at very large L we see only the lowest mass transverse "Goldstone modes" of the string leading to the classic string oscillator spectrum,

$$
E_{n}=T_{0} L+\frac{\pi}{L}\left[\sum_{i} a_{i}^{\perp \dagger} a_{i}^{\perp}-\frac{(D-2)}{24}\right]+\cdots
$$

Indeed at large separation $\mathrm{L}$ the lattice data for the stretched string spectrum appears to be approaching this form with 2 transverse oscillators, D-2 = 2. (See Fig. 6). Also using a very clever lattice simulation method, Lüscher and Weisz [16] were apparently able to determine the one loop contribution (i.e.the so called "Lüscher term") to the ground state,

$$
E_{0}^{1-l o o p}(L)=-\frac{\pi}{12}(1+0.12 f m / L),
$$

for $\mathrm{L}$ in the range 0.5 to $1.0 \mathrm{fm}$. The agreement with theory for $D-2=2$ is remarkable (if not paradoxical) in view of large non-universal features in the spectrum for $L \simeq 0.5-1.0 \mathrm{fm}$ clearly visible in the lattice data (Fig 6).

It should also be emphasized, contrary to the phrase "string limit?" on Fig. 6, for a fundamental string at large $N_{c}$ all distances scales must be described by quantized string. Moreover it is evident from the lattice data in Fig. 6 that a major challenge for the AdS/CFT approach to the QCD string is to understand the highly non-trivial interpolation between IR (large L) and UV (small L) physics of the string. As a first attempt, one may quantizing the string with Dirichlet boundary conditions in the confining $A d S^{5}$ black hole metric of Eq. 3.2 above and solve (numerically) for the minimal surface getting a classical potential energy, $E_{0}^{\text {class }}(L)$ for the ground state of the stretched string. This function obeys the following limits,

$$
E_{0}^{\text {class }}(L \rightarrow \infty) \simeq \frac{r_{\min }^{2}}{2 \pi \alpha_{s}^{\prime} R^{2}} L+O\left(L e^{-c L}\right) \quad \text { and } \quad E_{0}^{\text {class }}(L \rightarrow 0) \simeq-\frac{\pi^{2} \sqrt{2}}{\alpha_{s}^{\prime} \Gamma(1 / 4)^{4}} \frac{R^{2}}{L}
$$




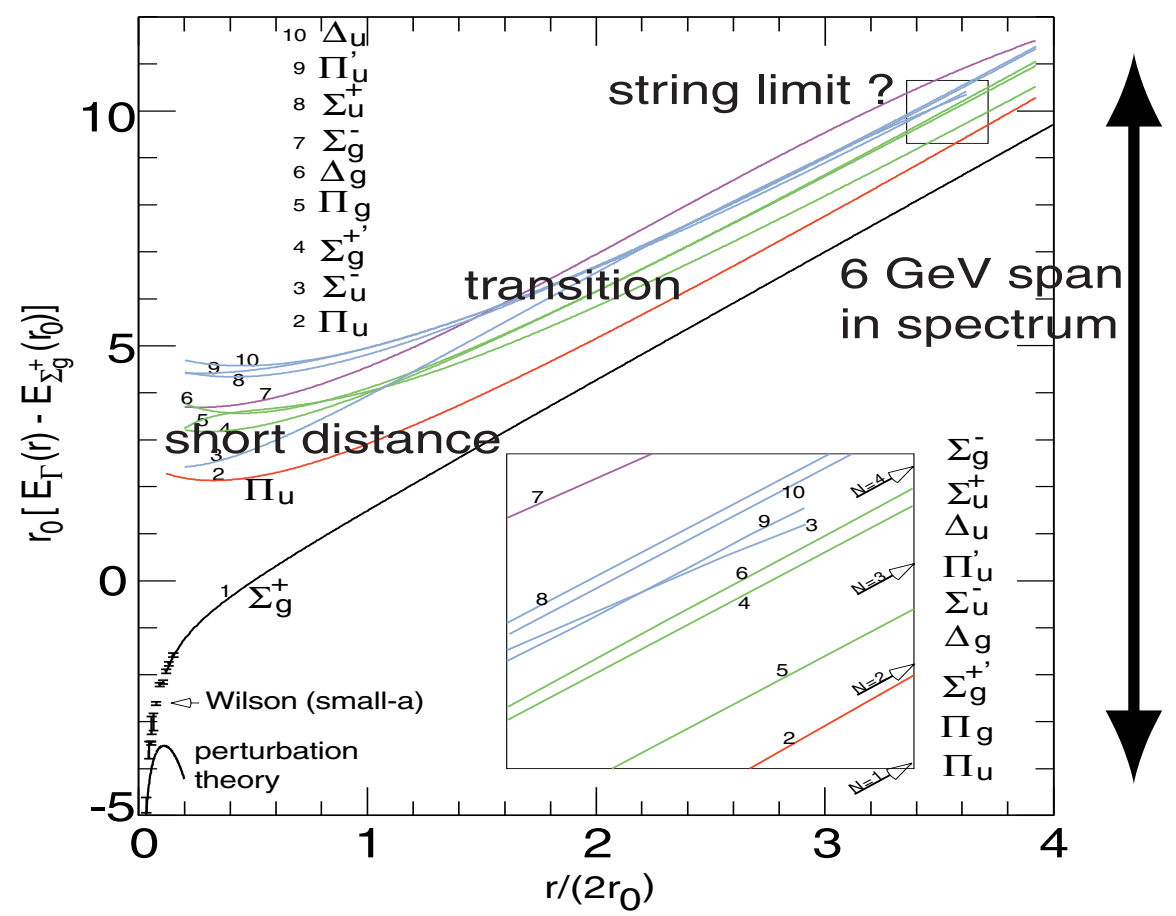

Figure 6: Quantum excitation spectrum of a stretch QCD flux tube calculated on the lattice by Juge, Kuti and Morningstar [14].

The exact function, $E_{0}^{\text {class }}(L)$, fits almost perfectly the lattice data for all $\mathrm{L}$, after adjusting the mass scale $R^{2} / r_{\min }=\Lambda_{I R}$ and the Regge slope $\alpha_{q c d}^{\prime}=R^{2} \alpha_{s}^{\prime} / r_{\min }^{2}$. This is reassuring but also highlights the present weakness of our AdS model. Pure Yang-Mills (quarkless QCD) predicts a definite number for the string tension, $T_{q c d}=1 /\left(2 \pi \alpha_{q c d}^{\prime}\right)$ relative to the single Yang-Mills scale, $\Lambda_{q c d}$. However at present all AdS/CFT strong coupling models for confining theory have an extra IR cutoff parameter that can be adjusted independent of the Regge slope (or QCD string tension). This extra mass scale is similar to strong coupling lattice QCD, except that on the lattice the new scale is the UV cutoff of the lattice spacing whereas here it is a phenomenological IR scale more similar to the MIT bag constant.

Any confining 5-d warped background (such as the AdS Black hole metric consider above) can be parameterized by $d^{2} s=R^{2}\left[d x^{2}+G^{2}(z) d z^{2}\right] / z^{2}$ where $r=R^{2} / z$. In this background a general wave equation for the transverse oscillations can be found in the semi-classical limit and solved for excitation energies, $E_{n}=E_{0}^{\text {class }}+\Delta E_{n}$, above the ground state, $E_{0}^{\text {class }}(L)$. In a temporal gauge $X^{(0)}=t$ with $\sigma=X^{(3)}$, the linearized or semi-classical equation for the transverse fluctuations of 
a string stretched is

$$
\left[\partial_{t}^{2}-v^{2}(\sigma) \partial_{\sigma}^{2}\right] X^{\perp}(t, \sigma)=0 .
$$

The local velocity, $v(\sigma)=z^{2}(\sigma) / z_{c}^{2}$, of propagation along the string is bounded by the speed of light. Here $z(\sigma)$ is the classical solution of the stretched string and $z_{c}$ is the maximum value of $z(\sigma)$ at the turning point in the middle of the string. At large L, the velocity approaches a constant, exponentially close to the speed of light, except near the quarks at $z=0$. Thus, it is not surprising that at large $\mathrm{L}$ this confining background reproduces flat string results,

$$
\Delta E_{n}=\frac{n \pi}{L},
$$

and in one loop, it reproduces the universal Lüscher term (or Casimire energy), $\Delta E_{n}^{1-l o o p}=-(D-$ 2) $\pi / 24 L$. Moreover a numerical solution to our equation in Fig. 7 shows deviations from the large $\mathrm{L}$ asymptotic value in qualitative agreement with that measured in the Lattice simulations of Juge, Kuti and Morningstar [14]. However there is another contribution to this deviation with the same

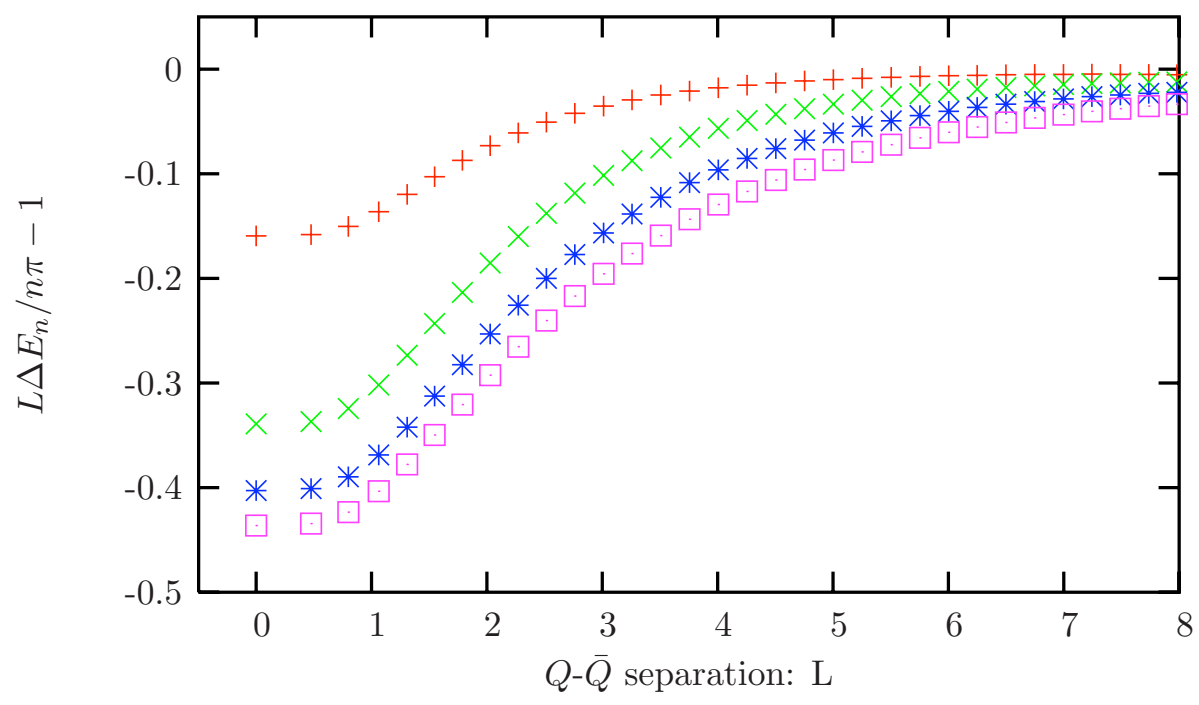

Figure 7: Deviations of transverse energy levels, $L \Delta E_{n} /(n \pi)-1$, in confining $A d S^{5}$ black hole model for $\mathrm{n}$ $=1(+), 2(\times), 3(*)$ and $4(\square)$.

sign observed in the exact spectrum of the (flat space) Nambu-Goto

$$
E_{n}^{\text {Nambu-Goto }}=T_{0} L \sqrt{1+\frac{2 \pi}{L}\left[\sum_{i} a_{i}^{\perp \dagger} a_{i}^{\perp}-\frac{(D-2)}{24}\right]}
$$

when quantized in the light-cone gauge [17]. An effort is underway to combine these two effects in a self-consistent light-cone quantization of the string in a confining AdS space. 
As you approach zero separation $L \rightarrow 0$, the discrete spectrum of the stretched string is determined by the pure $A d S^{5}$ metric [18]. The exact spectrum is know in closed form through the sum rule,

$$
z_{c} \Delta E_{n} \sqrt{\left(z_{c} \Delta E_{n}\right)^{4}-1} \int_{0}^{1} \frac{d s}{\left[1+\left(z_{c} \Delta E_{n}\right)^{2}\right] \sqrt{\left(1-s^{2}\right)}}=\frac{n \pi}{2},
$$

give by Callan and Guijosa [19] where $n=1,2, \cdots$ and $z_{c}=(2 \pi)^{-3 / 2} \Gamma\left(\frac{1}{4}\right) L$. In the conformal limit the energies must of course be proportional $1 / L$.

In addition to transverse Goldstone modes, there are quantum modes for fluctuations in the extra "radial" direction $r$,

$$
\left[\partial_{t}^{2}-v^{2}(\sigma) \partial_{\sigma}^{2}\right] \rho(t, \sigma)=M^{2}(\sigma) \rho(t, \sigma) .
$$

Unlike the transverse Goldstone modes $X^{\perp}$, now there is a $\sigma$-dependent "rest mass",

$$
M^{2}(\sigma)=-\frac{1}{z_{c}^{4}}\left[z^{3} \frac{d}{d z} \frac{1}{G^{2}}+\frac{2 z^{2}}{G^{2}}\right]_{z=z(\sigma)},
$$

where $z(\sigma)$ is the minimal surface for the classical solution in the background metric. The mass scale is set by the glueball mass: $M_{G B} \sim 1 / z_{c}$. These modes correspond via the String/Gauge duality to longitudinal (breathing) modes for a fat chromodynamic flux tube [18]. It would be interesting to find these modes in lattice gauge theory simulations and trace their dependence on $\mathrm{L}$.

Still our toy QCD string in an $A d S^{5}$ black hole is at best just a first step in understanding how a QCD string in warped space might behave. Much work remains even to identify the microscopic degrees of freedom of the QCD string let alone to the discovery of an effective string action capable of reproducing the lattice spectrum from long distance into the short distance region governed by asymptotically free gauge theory at large $N_{c}$. However a reasonable near term goal is to find accurate interpolation formulae for all $\mathrm{L}$ for the low energy spectrum of the stretched sting in a legitimate confining super gravity background. On the basis of the comparison of this spectrum with lattice data one might narrow the search for the QCD background geometry itself.

\section{High Energy Scattering}

We know that QCD, even in leading order of large $N_{c}$, exhibit asymptotic freedom and hard parton scattering properties. Consequently for the QCD string, one of the most baffling features in flat space is the complete absence of hard scattering. One the other hand for the application of string theory to quantum gravity, this softening of the short distance physics is a virtue, which is responsible for a finite weak coupling limit. Here we explain a surprisingly simple mechanism to reconcile this apparent conflict for strings duals to Yang-Mills theory.

\subsection{Hard Scattering at Wide Angles}

Let us begin with a description of the fundamental "Rutherford experiment" for the scattering two hadrons at wide angles. It is well known that up to logarithmic corrections due the running coupling, QCD exhibits power law fall off at wide angles precisely due to hard (UV) processes

$$
A_{q c d}(s, t) \sim\left(\frac{1}{\sqrt{\alpha_{q c d}^{\prime} s}}\right)^{n-4},
$$


where $n=\sum_{i} \tau_{i}=\sum_{i}\left(d_{i}-s_{i}\right)$ is the sum over twists $\tau_{i}$ for external states. In stark contrast the fundamental strings (in flat space) exhibits exponentially damped wide angle scattering,

$$
A_{\text {closed }}(s, t) \rightarrow \exp \left[-\frac{1}{2} \alpha^{\prime}(s \ln s+t \ln t+u \ln u)\right] .
$$

Polchinski and Strassler [20] made the essential observation on how string scattering in a confining background AdS background avoids this conflict with QCD. Suppose you have a background that is cut-off for small $r<r_{\min }$ and approximated by $A d S^{5} \times W$ for large r,

$$
d s^{2}=\frac{r^{2}}{R^{2}} \eta_{\mu \nu} d x^{\mu} d x^{v}+\frac{R^{2}}{r^{2}} d r^{2}+d s_{W}^{2} .
$$

A plane wave external glueball, $\phi(r) \exp [i x p]$, at strong coupling scatters locally in $\mathrm{r}$ through a string amplitude with a red shifted proper distance or equivalently an effective momenta,

$$
\hat{p}_{s}(r)=\frac{R}{r} p
$$

Relative to the string scale, $l_{s}=\sqrt{\alpha^{\prime}}$, the exponential cut-off at high momenta $\left(l_{s} p_{s}>1\right)$, suppresses string scattering in the IR region $\left(r<r_{\text {scatt }}\right)$, leaving a residual amplitude in a decreasingly small window in the $\mathrm{UV}\left(l_{s} p_{s}<1\right)$,

$$
r>r_{\text {scat }} \equiv \sqrt{\alpha^{\prime}} R p .
$$

Since the tail of the glueball wave function, $\phi_{i}(r) \sim\left(r / r_{\text {min }}\right)^{-\Delta_{4}^{(i)}}$, is entirely determined in the String/Gauge dictionary by the conformal weight $\Delta_{4}^{(i)}$ of the corresponding gauge operator dual to the string state, one is led back to the standard parton or naive dimensional analysis result used in the wide angle power counting rule,

$$
\phi_{i}\left(r_{s c a t}\right) \sim\left(\frac{r_{s c a t}}{r_{\text {min }}}\right)^{-\Delta_{4}^{(i)}} \sim\left(\sqrt{\alpha_{q c d}^{\prime}} p\right)^{-\Delta_{4}^{(i)}}
$$

where we have converted to the hadronic scale,

$$
\alpha_{q c d}^{\prime} \sim\left(R / r_{\min }\right)^{2} \alpha^{\prime}
$$

In the corresponding M-theory construction (sometime referred to as M-QCD), all of this appears to be upset because the scaling of the wave function in $A d S^{7}$ changes. For example the scalar glueball with interpolating field $\operatorname{Tr}\left[F^{\mu v} F_{\mu v}\right]$ in $A d S^{5}$ has $\Delta_{4}=4$ as expected but in $A d S^{7}$ the wave function scales with $\Delta_{6}=6$ at large $\mathrm{r}$. As pointed out by Brower and Tan [21], this apparent conflict with partonic expectations is avoided when one realizes that from an M-theory perspective, strings are a consequence of membranes wrapping the 11th dimension and that in $A d S^{7}$ this 11th dimension is warped just like another spatial coordinate $\left(x^{\mu}\right)$ with the proper size: $\hat{R}_{11}(r)=(r / R) R_{11}$. To account for this effect, one can introduce local values for the effective string length and coupling constant,

$$
\hat{l}_{s}^{2}(r)=\frac{R}{r}\left(l_{p}^{3} / R_{11}\right), \quad \text { and } \quad \hat{g}_{s}^{2}(r)=\frac{r^{3}}{R^{3}}\left(R_{11}^{3} / l_{p}^{3}\right) .
$$

as a function of the local scattering position in $\mathrm{r}$. This additional deformation is precisely what is required. The new definition of the scattering region at wide angles,

$$
r>r_{\text {scat }}=\hat{l}_{s}\left(r_{\text {scat }}\right) R p=\sqrt{\alpha^{\prime}} R^{\frac{2}{3}} r_{\text {scat }}^{-\frac{1}{2}} p
$$


leads to

$$
\phi_{i}\left(r_{s c a t}\right) \sim\left(\frac{r_{s c a t}}{r_{\text {min }}}\right)^{-\Delta_{6}^{(i)}} \sim\left(\sqrt{\alpha_{q c d}^{\prime}} p\right)^{-\frac{2}{3} \Delta_{6}^{(i)}}
$$

for each external line. For example, for the $0^{++}$scalar glueball corresponding to interpolating YM operator $\operatorname{Tr}\left[F^{2}\right]$, the factor of $2 / 3$ exactly compensates for the the shift in the conformal dimension from $\Delta_{4}=4$ for $A d S^{5}$ to $\Delta_{6}=6$ for $A d S^{7}$ to give the parton results, $n_{i}=\frac{2}{3} \Delta_{6}^{(i)}$. This time, in converting to the hadronic scale in Eq. 6.6, we must realize the relationship of $\alpha_{q c d}^{\prime}$ to the string scale is

$$
\alpha_{q c d}^{\prime} \sim\left(R / r_{\min }\right)^{3} \alpha^{\prime}
$$

which differs from the $A d S^{5}$ string relation (6.5). The 3 rd power is a consequence of the fact that in M-theory the area law for the Wilson loop really comes from a minimal volume for a wrapped membrane world volume stabilized at $r \simeq r_{\text {min }}$ rather than a minimal world surface area for a string which gave quadratic behavior in Eq. 6.5 .

Putting all factors together, the result for M-theory can be expressed as,

$$
\Delta \sigma_{2 \rightarrow m} \simeq \frac{1}{S} f\left(\frac{p_{i} \cdot p_{j}}{s}\right) \frac{1}{N_{c}^{2 m}} \prod_{i}\left(\frac{1}{\alpha_{q c d}^{\prime} S}\right)^{n_{i}-1},
$$

in agreement with the weak-coupling QCD results.

\subsection{Near-Forward Scattering and Regge Behavior}

The importance of scattering at large $r$ also suggests the presence of a hard component in the near-Regge limit, $t / s \rightarrow 0$ as $s \rightarrow \infty$. In Ref. [21] a heuristic argument was made as follows. The approximation of a single local scattering leads to $T(s, t)=\int_{r_{h}}^{\infty} d r \mathscr{K}(r) A(s, t, r)$, where $A$ is a local 4-point amplitude, $\mathscr{K}(r) \sim \phi_{1}(r) \phi_{2}(r) \phi_{3}(r) \phi_{4}(r)$ and $r_{h}$ is a high energy cut-off, $r_{h}>>r_{\text {min }}$. After converting to local string parameters as discussed above, the amplitude $A(s, t, r)$ depends only on $\alpha^{\prime} \hat{s}$ and $\alpha^{\prime} \hat{t}$, where $\hat{s}=(R / r)^{2} s$ and $\hat{t}=(R / r)^{2} t$ for the hardwall $A d S^{5}$ metric. In the Regge limit the amplitude becomes

$$
T(s, t)=\int_{r_{h}}^{\infty} d r \mathscr{K}(r) \beta(\hat{t})\left(\alpha^{\prime} \hat{s}\right)^{\alpha_{0}+\alpha^{\prime} \hat{t}} .
$$

For small $t \simeq 0$, this corresponds to an exchange of a BFKL-like Pomeron, with a small effective Regge slope,

$$
\alpha_{B F K L}^{\prime}(0) \sim\left(r_{\min } / r_{h}\right)^{2} \alpha_{q c d}^{\prime}<<\alpha_{q c d}^{\prime} .
$$

Such an exchange naturally leads to an elastic diffraction peak with little shrinkage. In the coordinate space, one finds, for a hard process, the transverse size is given by

$$
\left\langle\vec{X}^{2}\right\rangle \sim\left(r_{\min } / r_{h}\right)^{2} \alpha_{q d d}^{\prime} \log s+\text { constant } .
$$

If the cutoff, $r_{h}$, which characterizes a hard process, increases mildly with $s$, e.g. $r_{h}^{2} \sim \log s$, there will be no transverse spread. In the language of a recent study by Polchinski and Susskind, [22], this corresponds to "thin" string fluctuation. 
In spite of this progress in seeing some hard scattering effects in the string picture, there is much more to understand. For instance, we note that, consistent with the known spectrum of glueballs at strong coupling, the IR-region must in addition give a factorizable Regge pole contribution,

$$
T(s, t) \sim A\left(s, t, r_{\text {min }}\right) \sim\left(\alpha_{q c d}^{\prime} s\right)^{\alpha_{P}(0)+\alpha_{q c d}^{\prime} t} .
$$

where $\alpha_{P}(0)=2-0\left(1 / \sqrt{g^{2} N_{c}}\right)$. Of course, this "soft" Pomeron must mix with the corresponding hard component, leading to a single Pomeron singularity in the large $N_{c}$ limit. As emphasized by Polchinski and Strassler in a recent paper [23], this is also what is required for treating deep inelastic scattering in the string/gauge duality picture. This more careful analysis, which has been carried out recently by Brower, Tan, Polchinski and Strassler [24], will be presented next.

\section{BFKL Pomeron at Strong Coupling}

One of the most striking aspects of high-energy hadronic scattering is the continued increased of the total cross section, $\sigma_{T}$, with the energy. In a Regge language, this requires a leading j-plane singularity ${ }^{1}$ with vacuum quantum numbers having an intercept above $j=1$, in the forward limit, $t=0$. It has been a long held belief that QCD in the large $N_{c}$ limit, should be described by a stringlike theory. In such a framework, this leading singularity, a.k.a., Pomeron, should correspond to the leading closed string excitations in the crossed channel. There are currently two seemingly conflicting interpretations of high energy near-forward collisions in QCD: One based on perturbative leading log approximation (LLA), i.e., the BFKL Pomeron, and another based on nonperturbative (large- $N_{c}$ and/or phenomenological) considerations, i.e. the Soft Pomeron. Whereas the leading BFKL Pomeron represents LLA of gluon ladder graphs in the conformal limit, the dynamics of a soft Pomeron should be tied to confinement dynamics, e.g. glueball spectrum.

Recent development in AdS/CFT duality has provided new theoretical impetus for re-examining this issue. The Maldacena conjecture [1] and its further extensions [12, 25] state that Yang-Mills theory is exactly dual to a critical string theory in a non-trivial gravitational background. The particular target Yang-Mills theory depends on the geometry and symmetries of the string/gravity dual. By an appropriate choice, one is led to a specific suggestion as to how quarkless QCD (or $S U\left(N_{c}\right)$ Yang Mills theory) may be represented by a theory of closed strings. To regain approximate conformal invariance at short distances, the target space geometry should be asymptotically approximated by $A d S^{5}$ in the UV.

Rather than treating perturbative and non-perturbative frameworks separately, I will describe how the AdS/CFT duality provides a natural synthesis of both approaches by considering Regge behavior for bosonic string interaction in warped space time. The resulting j-plane structure is robust, which can be schematically represented as in Fig. 8. Under a Gaussian approximation, we identify Regge behavior as simultaneous random walk in transverse space, both the flat impact space and the AdS space, with confinement deformation. In particular, conformal invariance allows us to compute the BFKL intercept in the strong coupling limit. We begin in the next section by briefly reviewing key features of the perturbative Pomeron (BFKL) and the non-perturbative (soft)

\footnotetext{
${ }^{1}$ Of course to obey the Froissart bound, this j-plane singularity at leading order in the $1 / N_{c}$ perturbation expansion must eventually be hidden by unitarity corrections which dominate at even higher energies.
} 


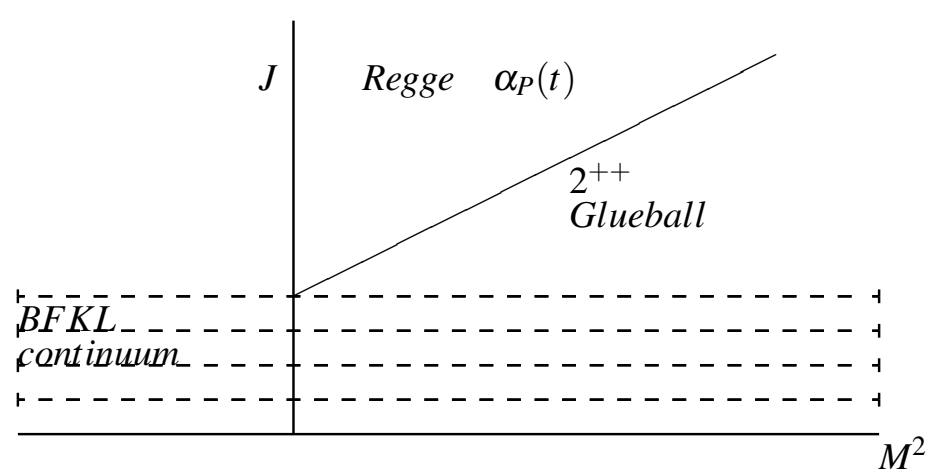

Figure 8: Soft Pomeron Regge pole at $J=\alpha_{P}(t)$ terminates at the BFKL cut with intercept at $J=\alpha_{B F K L}(0)$

Pomeron, emphasizing both their differences and similarities. We next show how these key features can be unified in a curved-space string theory in a light-cone description.

\subsection{Diffusion in Impact Space and Virtuality}

Due to a linear confining potential, QCD spectrum in the large $N_{c}$ limit contains states with arbitrarily high spin and masses, lying on nearly parallel Regge trajectories. It is also expected that, in the near forward limit at high energy, scattering amplitudes exhibit Regge behavior, e.g. for $2 \rightarrow 2$ scattering amplitudes, $\mathscr{A}(s, t) \sim \beta(t) s^{\alpha(t)}$, where $\alpha(t)$ and $\beta(t)$ are the Regge trajectory and residue respectively. It is well-known that these features can best be illustrated by string amplitudes in flat space.

However, by summing certain set of perturbative diagrams in QCD, for scattering with $s \gg$ $-t \gg \Lambda_{Q C D}$ (small fixed angles and ultra-high energies), Balitsky and Lipatov [27] and of Fadin and Kuraev [26] have obtained a different result, referred to as "BFKL". Extending their analysis to the forward limit, an elastic amplitude takes on the form $\int \frac{d k_{\perp}}{k_{\perp}} \int \frac{d k_{\perp}^{\prime}}{k_{\perp}^{\prime}} \Phi_{1}\left(k_{\perp}\right) K\left(s ; k_{\perp}, k_{\perp}^{\prime}\right) \Phi_{2}\left(k_{\perp}^{\prime}\right)$. Each of the two functions $\Phi_{i}$, called an "impact factor", describes the transverse structure of the corresponding hadronic state. These impact factors are convoluted together with the BFKL kernel, which can be computed exactly $[27,26]$ (in the BFKL leading-log approximation). A good approximation to this kernel leads to

$$
K\left(s, k_{\perp}, k_{\perp}^{\prime}\right) \approx \frac{s^{\omega_{0}}}{\sqrt{\pi \ln s}} e^{-\left[\left(\ln k_{\perp}^{\prime}-\ln k_{\perp}\right)^{2} / 4 \mathscr{D} \ln s\right]}
$$

where $\omega_{0}=4 \ln 2 \lambda / \pi$, and $\mathscr{D}=14 \zeta(3) \lambda / \pi$. We may recognize $K$, in this approximation, as a diffusion kernel, with the diffusion occurring in the variable $\ln k_{\perp}$ over a diffusion time $\sim \ln s$. In this context, $k_{\perp}$ is often referred to as "virtuality". Note that the kernel is invariant under scale transformations, $k_{\perp} \rightarrow \lambda k_{\perp}$.

Classic Regge behavior, $\mathscr{A} \sim s^{\alpha(t)}$, is also known to be related to diffusion in transverse impact space. For a linear trajectory near $t=0, \alpha(t) \simeq \alpha_{0}+\alpha^{\prime} t$; a Regge amplitude, after transforming into impact space, also takes a diffusive form,

$$
\int d^{2} k_{\perp} e^{i \mathbf{k}_{\perp} \cdot \mathbf{x}_{\perp}} s^{\alpha(t)}=s^{\alpha_{0}} \int d^{2} k_{\perp} e^{i \mathbf{k}_{\perp} \cdot \mathbf{x}_{\perp}} e^{-\alpha^{\prime} k_{\perp}^{2} \ln s} \sim \frac{s^{\alpha_{0}} e^{-x_{\perp}^{2} / 4 \alpha^{\prime} \ln s}}{\alpha^{\prime} \ln s} .
$$


Representing this by a Regge kernel in transverse impact space, it satisfies a diffusion equation:

$$
\left[\partial_{y}-\alpha_{0}-\alpha^{\prime} \partial_{x^{\perp}}^{2}\right] K\left(y ; x^{\perp}, x^{\prime \perp}\right)=\delta^{2}\left(x^{\perp}-x^{\perp}\right) \delta(y),
$$

where the evolution parameter is again the rapidity, $y \sim \log \left(\alpha^{\prime} s\right)$.

The similarity between these two types of diffusion is not accidental. As we will show, the kernel of BFKL, in the quadratic diffusive approximation Eq. (7.1) and in the appropriate kinematic regime, is reproduced in structure by the Regge behavior of string theory on a space of the form $A d S_{5} \times W$, where $A d S_{5}$ is five-dimensional anti-de Sitter space, and $W$ is a five-dimensional compact manifold that will play no role in the computation. In other words, the classical Regge behavior and the BFKL's result both emerge as features of Regge physics of a string theory compactified to $A d S_{5}$, in the form of simultaneous diffusion along the flat impact space and the curved fifth dimension.

To illustrate the key feature, consider scattering of hadrons of small size, i.e. hadrons built from constituents with mass much larger than the confinement scale $\Lambda$, correspond to modes of fields in the string theory with wave functions that have support only for $r \gg r_{\min }$, that is, in the asymptotically $A d S_{5}$ regime. For a pure $A d S_{5}$ background, the scattering of these objects for $t<0$ can be shown to be given by a Regge amplitude, in which the two wave functions are convoluted with a kernel that implements the Regge diffusion, but with the coordinate $x_{\perp}$ replaced with a function of the $A d S_{5}$ radial coordinate. As we will show, this function is $\ln r$. The resulting kernel is

$$
K\left(s ; r, r^{\prime}\right)=\frac{s^{\omega_{0}}}{\sqrt{\pi \ln s}} e^{-\left[\left(\ln r^{\prime}-\ln r\right)^{2} / 4 \mathscr{D} \ln s\right]}
$$

where $\omega_{0}=2-2 / \sqrt{\lambda}$, and $\mathscr{D}=1 / 2 \sqrt{\lambda}$. Comparing this with Eq. (7.1), one sees that the fifth coordinate $r$ of the string theory should be identified in this context with $k_{\perp}$ of the gauge theory. The identification of $r$ and $k_{\perp}$ has its source in the UV/IR correspondence and has been suggested in numerous contexts. In the next section, we will derive these results using string in curved background using light-cone gauge.

\subsection{Regge Behavior in light-cone gauge}

The light-cone has proven to be a natural formalism for studying the high energy limits of quantum field theories, leading to a vivid physical picture in Feynman's parton language. The lightcone gauge for a superstring [28] in $A d S^{5} \times W$ eliminates all spurious degrees of freedom in favor of the 8 transverse bosonic fields $X_{\perp}(\sigma, \tau)=\left(X_{1}, X_{2}\right), Y(\sigma, \tau)$ and the center of mass coordinates $x^{-}$and $p^{+}$, plus the corresponding Fermionic modes for the superstring. The light-cone gauge fixes $X^{+}(\sigma, \tau) \equiv\left(X^{0}+X^{3}\right) / \sqrt{2}=\tau, P^{+}(\sigma, \tau)=$ const, and the first derivatives of $X^{-}(\sigma, \tau)=$ $\left(X^{0}-X^{3}\right) / \sqrt{2}$ as quadratic functions of the transverse fields via the Virasoro constraints. (See Polchinski [11] and Zwiebach [29] for details.) Scattering amplitudes can now be defined in terms of light-cone path integrals. As an example, an elastic scattering amplitude $\left(p_{1}, p_{3} \rightarrow-p_{2},-p_{4}\right)$, is given by

$$
\mathscr{A}(s, t) \delta^{2}\left(p_{1}^{\perp}+p_{2}^{\perp}+p_{3}^{\perp}+p_{4}^{\perp}\right)=\mathscr{N} \int d T \int \mathscr{D} X_{\perp} D Y G^{1 / 2}[Z] V_{1} V_{2} V_{3} V_{4} e^{-\int d \tau \int_{0}^{p^{+}} d \sigma \mathscr{L}\left[X_{\perp}, Z\right]}
$$


where for the AdS direction we have set $Z(\sigma, \tau)=1 / R(\sigma, \tau)$ and ignored the bosonic modes in $W$ as well as all fermions. Scattering takes place on the world sheet illustrated in Fig. 9, with Neumann (open) or periodic (closed) boundary condition on the edges. The modulus $T$ is the time $\tau$ in the interaction region. (Closed strings have an additional modulus to enforce level matching.)

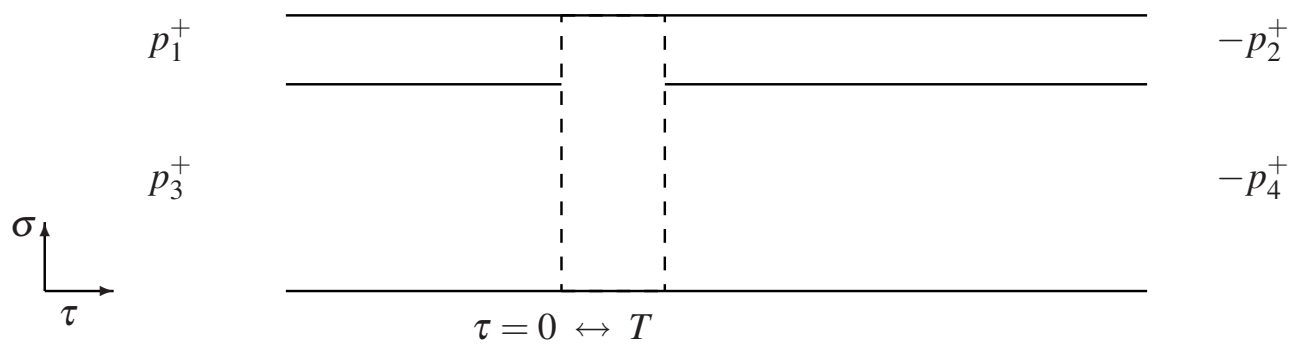

Figure 9: The light-cone world sheet domain, $X^{+}=\tau \in[-\infty, \infty], \sigma \in\left[0, p^{+}\right]$, with $p^{+}=p_{1}^{+}+p_{3}^{+}$for elastic scattering in the brick wall frame.

\subsection{Open string scattering in flat-spacetime}

The physical picture of Regge scattering in the light-cone frame can be appreciated with the simple example of open string elastic tachyon scattering,

$$
\mathscr{A}(s, t)=\int_{0}^{1} d w(1-w)^{-2-\alpha^{\prime} s} w^{-2-\alpha^{\prime} t} \simeq \Gamma\left(-1-\alpha^{\prime} t\right)\left(e^{-i \pi} \alpha^{\prime} s\right)^{1+\alpha^{\prime} t} .
$$

The Regge limit is dominated by the integration region where $w=O\left(1 / \alpha^{\prime} s\right)$.

Let us begin with a qualitative description of how this Regge limit translates into light-cone language. Since in the light-cone gauge the density of $P^{+}(\sigma)$ is conserved, it is traditional for scattering processes to label each string segment (or bit) $\Delta \sigma$ by equal quanta $\Delta p^{+}$by choosing the string length to be $l_{s}=p^{+}$. Also this gauge is not manifestly Lorentz invariant so it is helpful to pick a definite frame. As illustrated in Fig. 9, we have chosen the brick wall frame in the center of momentum, where the transverse momenta are reflected by the collision: $p_{r}^{\perp}= \pm k^{\perp} / 2$. In this frame strings join and split at exactly ${ }^{2}$ the same string bit $\left(\sigma=\sigma_{\text {int }}\right)$. The Regge limit represents the collision of a boosted "long" string that grow in length $\left(p_{3}^{+}=-p_{4}^{+} \sim O(\sqrt{s})\right)$ with a "short" string of decreasing length $\left(p_{1}^{+}=p_{2}^{+}=O(1 / \sqrt{s})\right)$. As a consequence the essential contribution involves a small area centered at the interaction region with $T \sim \Delta \tau \sim 1 / p_{3}^{+} \sim 1 / \sqrt{s}$ and $\Delta \sigma \sim$ $p_{1}^{+} \sim 1 / \sqrt{s}$. So one should be able to identify the Regge mechanism with a"local" conformal world sheet transformation near the interaction points.

With $T=0(1 / \sqrt{s})$, we may visualizing cutting the world sheet through the interaction region into two parallel horizontal strips. The sole impact of this brief interaction is for the ends of the

\footnotetext{
${ }^{2}$ To be precise, we define the brick wall frame with transverse momenta, $p_{1}^{\perp}=p_{2}^{\perp}=2 k^{\perp}$ and rapidities, $\exp \left[ \pm y_{i}\right]=$ $\sqrt{2} p_{i}^{ \pm} / \sqrt{\left(M_{i}^{2}+k_{\perp}^{2} / 4\right)}$ so that the invariants are, $t=-k_{\perp}^{2} \quad, \quad s=M_{1}^{2}+M_{3}^{2}+\sqrt{M_{1}^{2}+k_{\perp}^{2} / 4} \sqrt{M_{3}^{2}+k_{\perp}^{2} / 4} \cosh \left(y_{1}-\right.$ $\left.y_{3}\right)$. Boosting to the center of longitudinal momentum frame sets $y_{1}=-y_{3}$. In the brick wall frame the t-channel worldsheet diagram $(T<0)$ vanishes identically leaving only the s-channel contribution $(T \leq 0)$.
} 


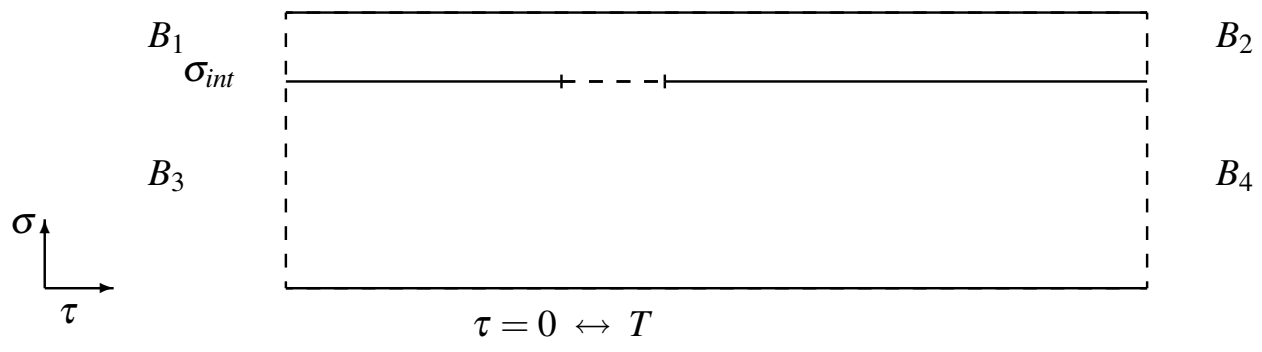

Figure 10: The light-cone world sheet domain split into two parallel sheets at the dotted lines with $\tau \in[0, T]$ and $\sigma=\sigma_{i n t}$. Solid/dotted lines have Neumann/Dirichlet boundary respectively.

strings to be constrained to coincide for $\tau \sim 0$. This can be imposed by replacing the Neumann boundary condition by a Dirichlet boundary condition implemented with a delta-functional constraint for $\tau \in[0, T]: \delta\left(X_{\perp}^{\text {long }}\left(\sigma_{\text {int }}, \tau\right)-X_{\perp}^{\text {short }}\left(\sigma_{\text {int }}, \tau\right)\right)$. As we show below the Regge behavior comes entirely from the growth of the long string. To evaluate the light-cone path integral in Eq. 7.5 for flat space, we drop the $Z$ coordinate and introduce the light-cone Lagrangian density, $\mathscr{L}=\frac{1}{2} \dot{X}_{\perp}^{2}+\frac{1}{2} \frac{1}{\left(2 \pi \alpha^{\prime}\right)^{2}} \dot{X}_{\perp}^{2}$. Next we introduce the vertex functions for ground state tachyons on vertical boundaries $B_{r}$, in Fig. 10

$$
V_{r}\left[p_{r}, X\right]=e^{\left(1 / p_{r}^{+}\right) \int_{B_{r}} d \sigma\left[i p_{r}^{\perp} X_{\perp}\left(\sigma, \tau_{r}\right)+p_{r}^{-} X^{+}\left(\sigma, \tau_{r}\right)\right]},
$$

with center of mass coordinates, $\tau_{r}=\left(1 / p_{r}^{+}\right) \int_{B_{r}} d \sigma X^{+}$and $x_{r}^{\perp}=\left(1 / p_{r}^{+}\right) \int_{B_{r}} d \sigma X_{\perp}$. The limits $\tau_{r} \rightarrow \mp \infty$ for in/out states put the scattering amplitude on shell. Since both $p^{+}$and the Hamiltonian $P^{-}$are conserved, there is no need for a longitudinal delta-functions, $\delta^{2}\left(p_{1}^{ \pm}+p_{2}^{ \pm}+p_{3}^{ \pm}+p_{4}^{ \pm}\right)$. We are primarily interested in understanding diffusion in the transverse ("impact parameter") space. To this end we recognize that transverse momenta $p_{r}^{\perp}$ are conjugate to center of mass $x_{\perp}^{r}$ in impact space, and we integrate the path integral for the external states up to the interaction region, $\tau \in$ $[0, T]$, indicated by dotted lines in Fig. 9. The external states are "dressed" by Gaussian fluctuations, $V_{r} \rightarrow \Phi_{r}\left(X_{\perp}^{(r)}\right)=\exp \left[i p_{r}^{\perp} x_{\perp}^{(r)}-\frac{1}{2} \sum_{n=1}^{\infty} \omega_{n} X_{n}^{(r)} X_{n}^{(r)}\right]$, where we have adopted a plane wave expansion for $X_{\perp}(\sigma, \tau)$,

$$
X_{\perp}(\sigma, \tau)=x_{\perp}+i \frac{p_{\perp}}{p^{+}} \tau+\sqrt{\frac{2}{p^{+}}} \sum_{n=1}^{\infty} X_{n}(\tau) \cos \left(\omega_{n} \sigma / c\right) \quad, \quad X_{n}(\tau)=\frac{a_{n}^{\dagger} e^{-\omega_{n} \tau}+a_{n} e^{\omega_{n} \tau}}{\sqrt{2 \omega_{n}}},
$$

with frequencies $\omega_{n}=n /\left(2 \alpha^{\prime} p^{+}\right)$. The fact that frequencies decrease under a boost has the consequence that strings with large/small $p^{+}$appear to be more weakly/strongly bound.

The analogous expansion for Regge limit now takes the form of an expansion in the interaction time T. To zeroth order, string- 1 and string- 2 coincide and so do string- 3 and string- 4 , and the two separate strings momentarily join at a point with Dirichlet boundary condition, enforced by delta functions, $\delta^{2}\left(X_{\perp}^{(1)}(0,0)-X_{\perp}^{(3)}(0,0)\right)$, where we have re-labeled the interaction point by $\sigma=0, \tau=$ 0 . This allows the Regge limit of the amplitude to be written,

$$
\mathscr{A}(s, t) \delta^{2}\left(p_{1}^{\perp}+p_{2}^{\perp}+p_{3}^{\perp}+p_{4}^{\perp}\right) \simeq \int \frac{d^{2} k^{\perp}}{(2 \pi)^{2}} V_{12}\left(k^{\perp}\right) F_{34}\left(-k^{\perp}\right),
$$


in terms of "short" and "long" string factors $V_{12}$ and $F_{34}$. The expression is non-symmetric as a natural consequence of the "infinite momentum" frame.

For the "short" string, the excitation frequencies in the wave function grow forcing it to interact like a rigid point-like object, so that its center of mass and the interaction point effectively coincide. Consequently its vertex is local, $V_{12}(k) \sim(2 \pi)^{2} \delta^{2}\left(p_{1}^{\perp}+p_{2}^{\perp}-k^{\perp}\right)$. On the the other hand, for the "long" string, to first order in $\mathrm{T}$, the interaction reduces to $\frac{T}{2} \int_{0}^{p_{3}^{+}} d \sigma\left[\dot{X}_{\perp}^{2}+\frac{1}{\left(2 \pi \alpha^{\prime}\right)^{2}} X_{\perp}^{\prime}{ }^{2}\right]$. By expanding in normal modes, one finds

$$
F_{34}\left(-k^{\perp}\right) \simeq 2(2 \pi)^{2} \delta^{2}\left(p_{3}^{\perp}+p_{4}^{\perp}+k^{\perp}\right) \int d T T^{-2} p^{+} e^{-p^{-} T} \exp \left[-\sum_{n} \frac{\alpha^{\prime} k_{\perp}^{2}}{n+n^{2} T / 2 \alpha^{\prime} p_{3}^{+}}\right],
$$

where we have normalized the form factor by identifying with the "photon" pole contribution at $k^{\perp}=0$. The sum in the exponent, at large $p_{3}^{+} \simeq p^{+} \simeq s / 2 p^{-}$, after integrating over $\zeta=p^{-} T$, leads to the final result,

$$
\mathscr{A}(s, t) \simeq \Gamma\left[-1-\alpha^{\prime} t\right]\left(-\alpha^{\prime} s\right)^{\left[1-\alpha^{\prime} k_{\perp}^{2}\right]} .
$$

where $t=-k_{\perp}^{2}$.

We re-iterate the key advantage of the light-cone approach that allows one to interpret Regge behavior as a diffusion process in transverse (impact parameter) space. To do this consider the kernel in impact parameter space, $K\left(y ; x_{1}^{\perp}, x_{3}^{\perp}\right)$, which is just the Fourier transform of the $s$-dependent factor in Eq. 7.10. This satisfies the diffusions equation,

$$
\left[\partial_{y}-1-\alpha^{\prime} \partial_{x_{1}^{\perp}}^{2}\right] K\left(y ; x_{1}^{\perp}, x_{3}^{\perp}\right)=\delta\left(x_{1}^{\perp}-x_{3}^{\perp}\right) \delta(y),
$$

where evolution parameter is the rapidity, $y \sim \log \left(\alpha^{\prime} s\right)$. Indeed we could just as well have derived this diffusion equation directly from the path integral by working with transverse coordinates, taking the derivative with respect to rapidity. Not only does this give a physical picture of the Regge limit, this approach often has technical advantages in more complex target space geometries such as our example leading to the BFKL singularity. We see in flat space that the diffusive growth in impact parameter $\left\langle\left(x_{3}^{\perp}-x_{1}^{\perp}\right)^{2}\right\rangle \sim \log \left(\alpha^{\prime} s\right)$ causes what is known as "Regge shrinkage" of the Regge "form factor", $\exp \left[\alpha^{\prime} t \log \left(\alpha^{\prime} s\right)\right]$ with $t=-k_{\perp}^{2}<0$. The amplitude decreases more rapidly in $|t|$ at large values of the energy. Solution to the diffusion equation determines the leading Regge singularity.

\subsection{Regge behavior in warped spacetime}

The light-cone Lagrangian in AdS space [28] is

$$
L=\frac{1}{2} \int_{0}^{p^{+}} d \sigma\left[\dot{X}_{\perp}^{2}+\dot{Z}^{2}+\frac{1}{\left(2 \pi \alpha^{\prime} R_{a d s}^{-2} Z^{2}\right)^{2}}\left(X_{\perp}^{\prime 2}+Z^{\prime 2}\right)\right] .
$$

In the light-cone frame the conformal group $O(4,2)$ is restricted to the subgroup $S L(2, C)$, which includes $Z \rightarrow \lambda Z, X_{\perp} \rightarrow \lambda X_{\perp}, \tau \rightarrow \lambda \tau, \sigma \rightarrow \sigma / \lambda$. To exploit this we change variables to $U(\sigma, \tau)=$ $-\log \left(Z(\sigma, \tau) / R_{a d s}\right.$ and make a semi-classical expansions around the zero modes $U=u, X_{\perp}=$ $x_{\perp}$. The essential new feature is an effective string slope, $\alpha_{\text {eff }}^{\prime}(u)=\alpha^{\prime} e^{-2 u}$, which leads to local dependences on $u$. The (Gaussian) dressed wave functions become

$$
V_{r} \rightarrow \Phi_{r}[X, U]=e^{i p_{\perp}^{(r)} x_{\perp}^{(r)}} e^{-\frac{1}{2} \sum_{n} \omega_{n}\left[e^{2 u} X_{n}^{(r)} X_{n}^{(r)}+R_{a d s}^{2} U_{n}^{(r)} U_{n}^{(r)}\right]} \psi_{r}\left(u_{r}\right) .
$$


Again we make a first order approximation to the interaction term: $i p^{+} T \int_{0}^{p^{+}} d \sigma \dot{X}_{+}(\sigma, 0)$.

Factorization of the Dirichlet constraint on $U$ in the interaction region requires a new delta function,

$$
\delta\left(U_{\perp}^{(1)}(0,0)-U_{\perp}^{(3)}(0,0)\right)=\int \frac{d v}{2 \pi} \exp \left[i v\left(U^{(1)}(0,0)-U^{(3)}(0,0)\right)\right] .
$$

Again diffusion takes place only for the "long" boosted string. The details are analogous to the flat space example, except that at Eq. $7.9 \alpha^{\prime}$ is replaced by $\alpha_{e f f}^{\prime}(u)$ and a new term $v^{2}$ is added to $\alpha_{e f f}^{\prime}(u) k_{\perp}^{2}$. For $k_{\perp}^{2}=0$, the $v^{2}$ factor clearly corresponds to diffusion in the $u$-direction. For non-zero $k_{\perp}^{2} \neq 0$, we must be represented $v=i \partial_{u}$ as an operator conjugate to $u$.

However, to obtain the correct diffusion equation one must go to one loop order beyond the Gaussian approximation to the world sheet in the sigma model to find an anomalous shift $v^{2} \rightarrow$ $v^{2}+2 i v$. Here we choose to fixed this shift by matching with the on-mass- shell condition $\left(L_{0}=1\right.$ at $j=1$ ) for the vector field in AdS space. The result in any case is a Hermitian differential equation which specifies the leading $\mathrm{j}$-plane singularity,

$$
\left[\partial_{y}-1-\alpha^{\prime} e^{-2 u} \partial_{x}^{2}-\frac{1}{\sqrt{\lambda}}\left(\partial_{u}^{2}-1\right)\right] \mathscr{K}\left(y ; x, u, x^{\prime}, u^{\prime}\right)=\delta^{2}\left(x-x^{\prime}\right) \delta\left(u-u^{\prime}\right) \delta(y) .
$$

to leading order in $\alpha^{\prime}$. We note that diffusion in $u$ suppresses the corresponding diffusion in impact parameter space, giving rise to the BFKL cut. In fact this effect could have been anticipated qualitatively in terms of the boosted incoming wave function. The $U_{n}$ modes enter like ordinary transverse modes in flat space so diffusion naturally drives the incoming hadron into the UV (large $\mathrm{u}$ ), which then acts to increase the effective energies $\left(\omega_{n} e^{2 u}\right)$ of $X_{n}^{\perp}$ modes suppressing diffusion in impact parameter (see Fig. 11). This effect is counter to the flat space effect reducing $\omega_{n} \sim n / p^{+}$ which leads to Regge shrinkage. The result is a BFKL-like cut for $t<0$ for the open string Regge exchange starting at $j=1-1 / \sqrt{\lambda}$.

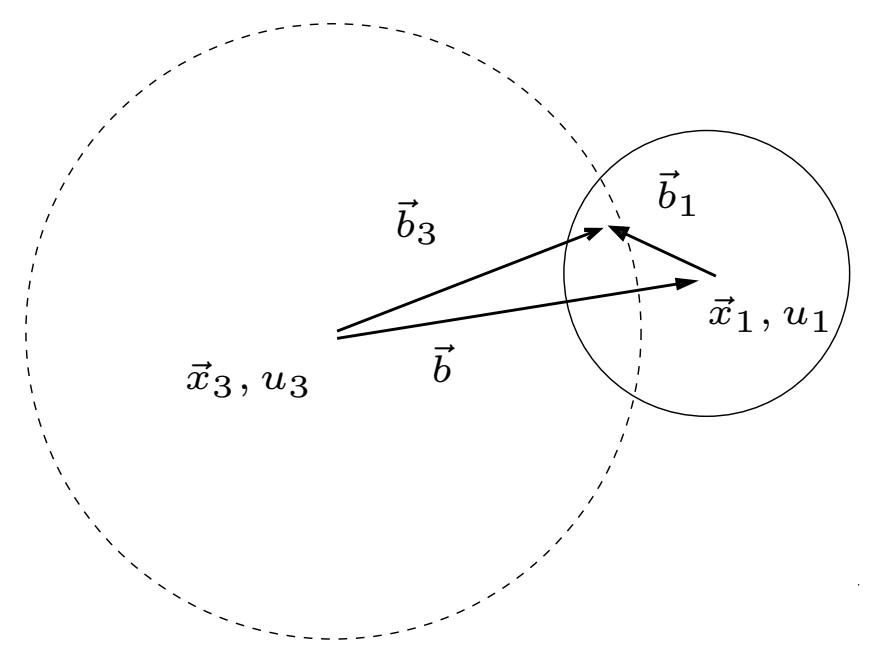

Figure 11: At the interaction the impact parameter is given by $\vec{b}=\vec{b}_{3}-\vec{b}_{1}$ where $\vec{b}_{i}$ is the vector from the center of mass of each string to its end point. In AdS space the strings are separated by an additional transverse co-ordinate $u=u_{1}-u_{3}=\ln \left(z_{3} / z_{1}\right)$ in the radial direction. 
Lastly, we can generalize this to the closed string by introducing periodic boundary condition on the strings and an additional modulus $\theta$ that rotates the Riemann surface around a cut on the s-channel intermediate closed string. Again the Gaussian approximation gives the Regge amplitude up to an anomalous correction. The integral over $\theta$ forces level matching between holomorphic and anti-holomorphic modes. This has the sole effect of replacing $\alpha^{\prime} \rightarrow \frac{1}{2} \alpha^{\prime}$. The final result, when transformed back to a $j-t$ representation, is

$$
\left.\left[j-2-\frac{\alpha^{\prime}}{2} t e^{-2 u}-\frac{1}{2 \sqrt{\lambda}}\left(\partial_{u}^{2}-4\right)\right)\right] \mathscr{K}\left(J, t ; u, u^{\prime}\right)=\delta\left(u-u^{\prime}\right) .
$$

For closed strings, we can also match in the strong coupling limit, at $j=2$, with the equation for tensor glueballs. Again we have corrected the Gaussian approximation by a comparison with the on-shell linearized graviton equation in the $A d S^{5}$ background by shifting $v \rightarrow v^{2}+4 i v$. Note that, in principle the anomalous shift mentioned above, noted by Gubser, Klebanov and Polyakov [25], could be computed by going to one loop order using the methods of Callan and Gan, [30]. It is interesting to note that Polchinski and Susskind [22] argue that this correction is essential to defining a finite graviton form factor at non zero $k_{\perp}^{2}$. Apparently the BFKL singularity, power behavior at wide angles [20,21] and finite power behaved form factors all have a common origin, at least in strong coupling conformal theories.

\subsection{Unified Soft and Hard Pomeron Model}

Strictly speaking the above derivation of the BFKL Pomeron in strong coupling is a result of pure conformal theory, so we are confident of the result for $\mathscr{N}=4$ super Yang Mills theory. However it is easy to modify the background metric to include qualitative effects of confinement in the IR and asymptotic scaling in the UV. The generic features are expected to apply to QCD. Spectrum in the j-plane (at strong coupling) is found by solving the Schrödinger equation,

$$
\left[-\partial_{u}^{2}+V(t, u)\right] \psi_{j}(u, t)=2 \sqrt{\lambda}(2-j) \psi_{j}(u, t),
$$

with the appropriately modified potential. For the hardwall model the conformal potential, $V(t, u)=$ $4-t \alpha^{\prime} \sqrt{\lambda} e^{-2 u}$, is modified by an appropriate boundary condition at the wall that restricting the wave function to $u>0$ (see Fig. 12 ). The result is the spectrum drawn in Fig. 13. Note that we now have a unified model for the hard (BFKL) and soft(Regge) Pomeron. For $t<0$ the continuum spectrum, namely the flat BFKL cut, gives the leading high energy contribution but at positive $t>0$ the potential develops bound state solution for Regge poles that account for the glueball spectrum at integer spin. If we modify the background in the UV to account for the running coupling, the BKFL continuum breaks up into a series of closely space Regge poles as illustrated in our strong coupling solution in Fig. 14. This effect has been suggested in the weak coupling limit as well. Two rather model independent observation can be make. (1) For large positive $t$, we have approximately linear trajectory $j \simeq \alpha^{\prime} t$. Only the rate of approach to linearity depends on the details of the confinement deformation. (2) For $|t|$ large and negative, the kernel is insensitive to confinement deformation and one finds a fixed BFKL singularity at $j=2-2 / \sqrt{\lambda}$. Finally in Ref. [24], it is noted that in a conformal theory the BFKL singularity appears to be determined by a single function in the conformal dimension/angular momentum plane $(\Delta-j$ in Fig. 15$)$. The BFKL intercept 


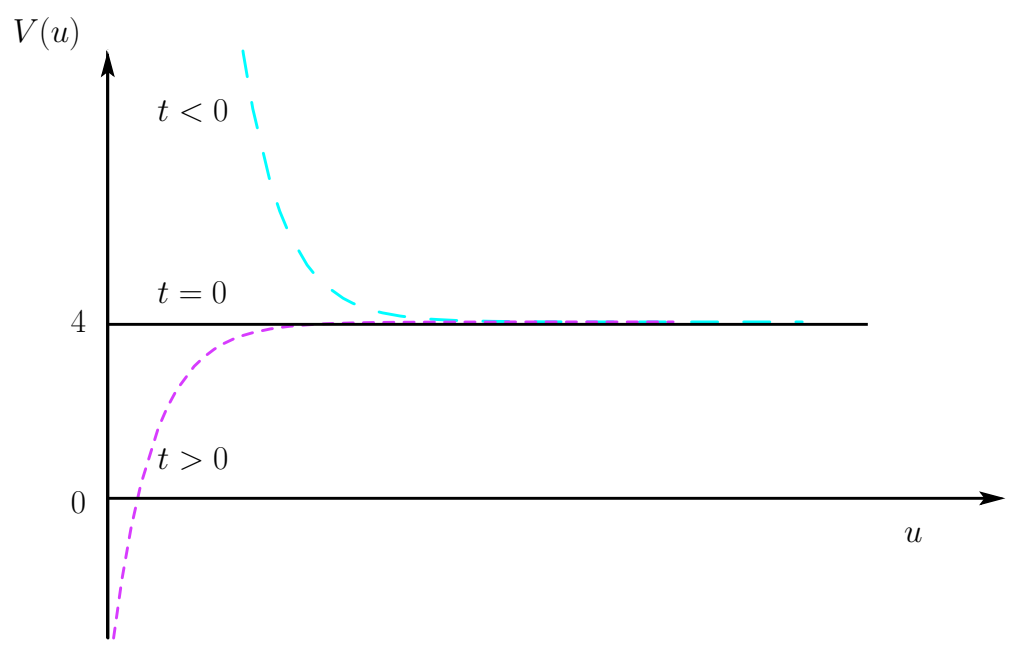

Figure 12: The potential for the effective Schrödinger problem in the hard-wall model, for $t=0$ (solid), $t>0$ (short dash), and $t<0$ (long dash).

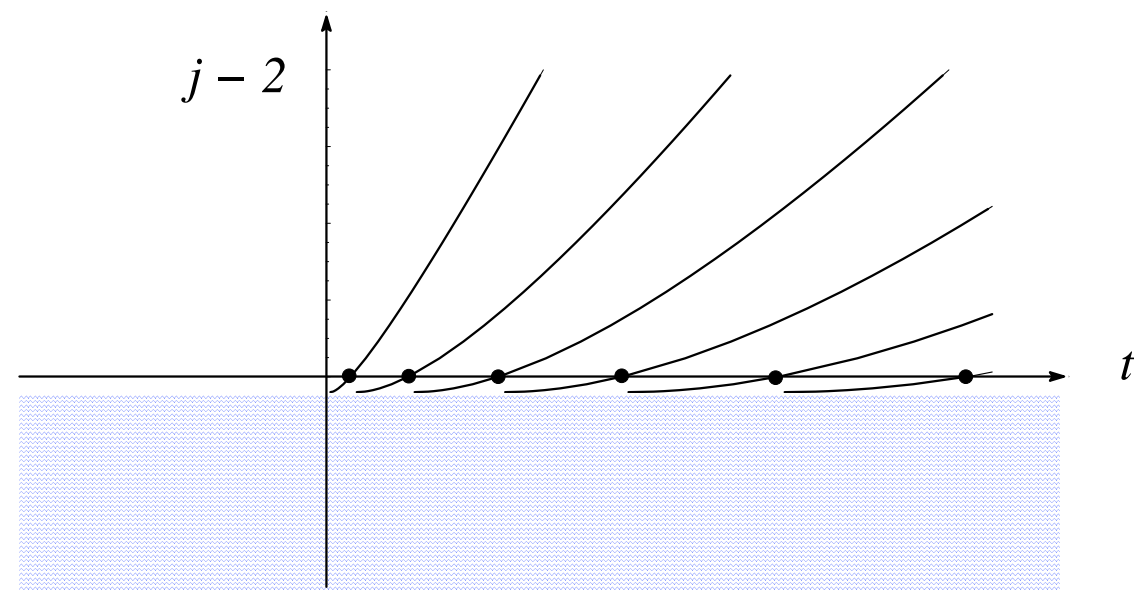

Figure 13: The analytic behavior of Regge trajectories in the hard-wall model, showing the location of the bound-state poles at $j=2$ and the $t$-independent continuum cut (shaded) at $j=j_{0}=2-2 / \sqrt{\lambda}$ into which the Regge trajectories disappear. The lowest Regge trajectory intersects the cut at a small positive value of $t$. At sufficiently large $t$ each trajectory attains a fixed slope, corresponding to the tension of the model's confining flux tubes.

is the minimum value of $\mathrm{j}$ as a function of $\Delta$, whereas the anomalous dimensions for moments of the deep inelastic structure functions (DGLAP) are given by values of $\Delta$ at integer $\mathrm{j}$. Understanding this connection in greater detail and applying it to QCD is an important task.

\section{Future Directions}

The construction of the QCD string theory remains a tantalizing but unrealized goal. Recent progress has certainly begun to show how such an exact string/gauge duality might arise. Indeed 


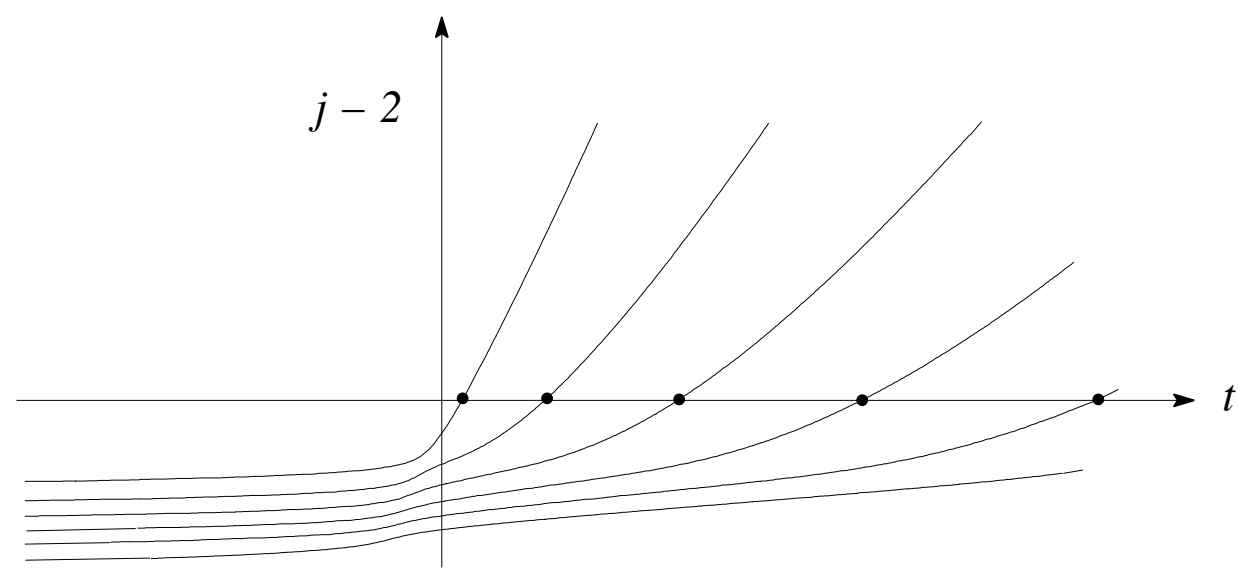

Figure 14: The analytic behavior of Regge trajectories with a running coupling in the UV and a hard-wall cut-off in IR.

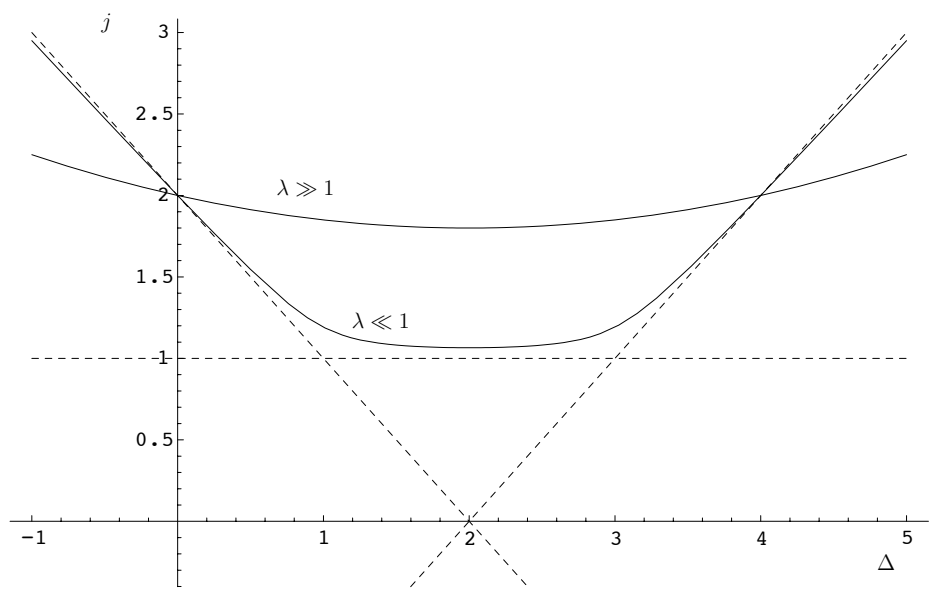

Figure 15: Schematic form of the $\Delta-j$ relation for $\lambda \ll 1$ and $\lambda \gg 1$. The dashed lines show the $\lambda=0$ DGLAP branch (slope 1), BFKL branch (slope 0), and inverted DGLAP branch (slope -1 ). Note that the curves pass through the points $(4,2)$ and $(0,2)$ where the anomalous dimension must vanish. This curve is often plotted in terms of $\Delta-j$ instead of $\Delta$, but this obscures the inversion symmetry $\Delta \rightarrow 4-\Delta$.

the intimate relations between Yang-Mills theory and string theory is a dramatic change in our understanding, which may aptly designated the "First String Counter Revolution" - bring the subject back to its earliest roots.

However I should also emphasize that in this short lecture, I was not able to include many interesting developments based on the AdS/CFT conjecture. From the perspective of lattice QCD, the two worst omissions concern (1) very interesting progress in understanding the open string sector (or probe quarks) in the context of the AdS/CFT and (2) qualitative insight into the quark gluon plasma being pursued experimentally in heavy ion collision. For the first problem the low energy limit of open strings gives a new insight into chiral symmetry breaking and the spectrum of low mass mesons $\pi, \rho, \ldots$ The realization of chiral symmetry in the $5^{\text {th }}$ dimension is amazingly 
similar to use of domain wall Fermions to implement exact chiral symmetry in lattice field theory. The dual to the Fermion fields are flavor "condensate" gauge fields on probe brains with right and left boundary in UV that carry the global $S U_{L}\left(n_{f}\right)$ and $S U_{R}\left(n_{f}\right)$ symmetries respectively. At present there is a quite successful bottom up construction of this sector as presented for example in Ref. [31]. The second problem has lead to a series of remarkable results such as the conjecture KSS entropy bound [32]. In addition the approach to high energy scattering discussed above is an active areas of research. Many questions remain such as unitarization and the mechanism for saturation of the Froissart bound at strong coupling as well as other non-perturbative effects in the coupling $g_{s} \sim 1 / N_{c}$ such as the giant graviton connection for the baryon and the role of instantons in the finite $N_{c}$ QCD vacuum.

At a fundamental level there is still much confusion on each of these topics with new ideas streaming forth. The most definitive mathematical progress is the top down approach based on tractable "toy models" of QCD with some residual supersymmetry or special limits where semiclassical methods can be applied. It must also be admitted that formidable challenges remain. Even for the simplest case of pure $A d S^{5} \times S^{5}$, it has not been possible to analytically quantize the free superstring. A basic problem remains to find a mechanism to really separate the charged KaluzaKlein state outside the QCD sector from the physical states that should survive at a QCD fixed point. Perhaps the framework of starting from a critical string is flawed. At present there is no a direct constructive method for defining the QCD string, ignoring the subsequent difficulty in solving it. This is in contrast with lattice gauge theory which is well defined in spite of the need to resort to numerical methods for its solution. Finally let me emphasize the opportunity of lattice gauge theory to contribute to narrowing the search for the QCD string and in the end giving definite tests of any conjectured solution. As an example in closing the gap between lattice methods and the QCD string is the numerical solution to $N_{c}=\infty$ QCD based on the Euguchi-Kawai matrix reduction being developed by Narayanan and Neuberger [33]. This gives a precise numerical method for computing important properties of the QCD string at $N_{c}=\infty$, which may provide invaluable data to discovering the relevant degrees of freedom of the QCD world sheet, traditionally the first step toward the discover of the quantum action. Once the world sheet fields are identified, one should proceed to effective world sheet theories to compare with the lattice data and in time hopefully to discover the correct action for the QCD string. We'll see.

\section{References}

[1] J. Maldacena, Adv. Theor. Math. Phys. (1998), 2:231

[2] S. R. Coleman, Phys. Rev. D 11, 2088 (1975).

[3] S. Mandelstam, Phys. Rev. D 11, 3026 (1975).

[4] R. C. Brower and J. Harte, “Kinematic Constraints for Infinitely Rising Regge Trajectories”, Phys. Rev. bf 164 (1967) 1841.

[5] G. Veneziano, "Construction of a crossing-symmetric Regge-behaved amplitude for linearly rising trajectories", Nuovo Cim 57A (1968) 190-197. To be precise this paper gave an amplitude for $\omega \rightarrow \pi^{+} \pi^{-} \pi^{0}$ which was extended to pion scattering by Lovelace and Shapiro shortly thereafter.

[6] R. Dolen, D. Horn and C. Schmid, "Finite Energy Sum Rules And Their Application To Pi N Charge Exchange,” Phys. Rev. 166, 1768 (1968). 
[7] A. Neveu and J. H. Schwarz, "Factorizable Dual Model Of Pions," Nucl. Phys. B 31, 86 (1971).

[8] G. 'tHooft, “A planar diagram theory for strong interactions”, Nuicl. Phys. B 72 (1974) 461-473.

[9] D. J. Gross and W. I. Taylor, Nucl. Phys. B 400, 181 (1993) [arXiv:hep-th/9301068].

[10] A. Armoni, M. Shifman and G. Veneziano, Phys. Rev. Lett. 91, 191601 (2003) [arXiv:hep-th/0307097].

[11] , J. Polchinski, "String theory. Vol. 1: An introduction to the bosonic string and Vol 2: Superstring theory and beyond", Cambridge, UK University. Press. (1998).

[12] E. Witten, Adv. Theor. Math. Phys. 2, 253 (1998) [arXiv:hep-th/9802150].

[13] C. J. Morningstar and M. Peardon, "The glueball spectrum from an anisotropic lattice study", Phys.Rev. D60 (1999) 034509.

[14] K. J. Juge, J. Kuti and C. Morningstar, Phys. Rev. Lett. 90, 161601 (2003) [arXiv:hep-lat/0207004].

[15] R. C. Brower, S. D. Mathur and C. I. Tan, Nucl. Phys. B 587, 249 (2000) [arXiv:hep-th/0003115].

[16] M. Luscher and P. Weisz, JHEP 0207, 049 (2002) [arXiv:hep-lat/0207003].

[17] J. F. Arvis, Phys. Lett. B 127, 106 (1983).

[18] R. C. Brower, C. I. Tan and C. B. Thorn, Phys. Rev. D 73, 124037 (2006) [arXiv:hep-th/0603256].

[19] C. G. . Callan and A. Guijosa, Nucl. Phys. B 565, 157 (2000) [arXiv:hep-th/9906153].

[20] J. Polchinski and M. Strassler "Hard scattering and gauge/string duality", Phys. Rev. Lett. 88, 031601 (2002)

[21] R. C. Brower and C-I Tan "Hard scattering in the M-theory dual for the QCD string" Nucl. Phys. B662,393-405 (2003)

[22] J. Polchinski and L. Susskind, "String theory and the size of hadrons," arXiv:hep-th/0112204.

[23] J. Polchinski and M. J. Strassler, JHEP 0305, 012 (2003) [arXiv:hep-th/0209211].

[24] R. C. Brower, J. Polchinski, M. J. Strassler and C. I. Tan, arXiv:hep-th/0603115.

[25] S. S. Gubser, I. R. Klebanov and A. M. Polyakov, Phys. Lett. B 428, 105 (1998) [arXiv:hep-th/9802109].

[26] E. A. Kuraev, L. N. Lipatov and V. S. Fadin, Sov. Phys. JETP 45, 199 (1977) [Zh. Eksp. Teor. Fiz. 72, 377 (1977)].

[27] I. I. Balitsky and L. N. Lipatov, Sov. J. Nucl. Phys. 28, 822 (1978) [Yad. Fiz. 28, 1597 (1978)].

[28] R. R. Metsaev, C. B. Thorn and A. A. Tseytlin, "Light-cone superstring in AdS space-time," Nucl. Phys. B 596, 151 (2001) [arXiv:hep-th/0009171].

[29] B. Zwiebach "A first course in string theory", Cambridge, UK: Univ. Pr. (2004)

[30] C. G. . Callan and Z. Gan, Nucl. Phys. B 272, 647 (1986).

[31] J. Erlich, E. Katz, D. T. Son and M. A. Stephanov, Phys. Rev. Lett. 95, 261602 (2005) [arXiv:hep-ph/0501128].

[32] P. Kovtun, D. T. Son and A. O. Starinets, "Viscosity in strongly interacting quantum field theories from black hole Phys. Rev. Lett. 94, 111601 (2005) [arXiv:hep-th/0405231].

[33] R. Narayanan and H. Neuberger, arXiv:hep-th/0607149. 